Digital Object Identifier (DOI) 10.1007/s10097-002-0044-y

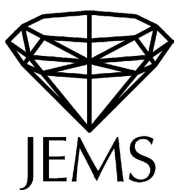

Burt Totaro

\title{
Complexifications of nonnegatively curved manifolds
}

Received December 15, 2000 / final version received April 17, 2002

Published online August 15, 2002 - (c) Springer-Verlag \& EMS 2002

Define a good complexification of a closed smooth manifold $M$ to be a smooth affine algebraic variety $U$ over the real numbers such that $M$ is diffeomorphic to $U(\mathbf{R})$ and the inclusion $U(\mathbf{R}) \rightarrow U(\mathbf{C})$ is a homotopy equivalence. Kulkarni showed that every manifold which has a good complexification has nonnegative Euler characteristic [16]. We strengthen his theorem to say that if the Euler characteristic is positive, then all the odd Betti numbers are zero. Also, if the Euler characteristic is zero, then all the Pontrjagin numbers are zero (see Theorem 1.1 and, for a stronger statement, Theorem 2.1). We also construct a new class of manifolds with good complexifications. As a result, all known closed manifolds which have Riemannian metrics of nonnegative sectional curvature, including those found by Cheeger [5] and Grove and Ziller [11], have good complexifications.

We can in fact ask whether a closed manifold has a good complexification if and only if it has a Riemannian metric of nonnegative sectional curvature. The question is suggested by the work of Lempert and Szóke [17]. Lempert and Szóke, and independently Guillemin and Stenzel [12], constructed a canonical complex analytic structure on an open subset of the tangent bundle of $M$, given a real analytic Riemannian metric on $M$. We say that a real analytic Riemannian manifold has entire Grauert tube if this complex structure is defined on the whole tangent bundle $T M$. Lempert and Szőke found that every Riemannian manifold with entire Grauert tube has nonnegative sectional curvature. Also, a conjecture by Burns [4] predicts that for every closed Riemannian manifold $M$ with entire Grauert tube, the complex manifold $T M$ is an affine algebraic variety in a natural way; if this is correct, the complex manifold $T M$ would be a good complexification of $M$ in the above sense. The problem remains open at this writing despite the paper of Aguilar and Burns [2], because the proof of Proposition 2.1 there is not yet generally accepted.

We can also ask how many Riemannian manifolds have entire Grauert tube. It is known to be a strong restriction: Szóke showed that of all surfaces of revolution diffeomorphic to the 2-sphere, only a one-parameter family of metrics of given volume have entire Grauert tube [22]. Aguilar showed that the quotient of a Riemannian manifold with entire Grauert tube by a group of isometries acting freely also has entire Grauert tube [1]. All known manifolds with entire Grauert

B. Totaro: DPMMS, Wilberforce Road, Cambridge CB3 0WB, UK, e-mail: b.totaro@dpmms.cam.ac.uk 
tube are obtained by Aguilar's construction: starting with a compact Lie group with a bi-invariant metric, or the product of such a group with Euclidean space, one takes the quotient by some group of isometries acting freely. That is striking because such quotient manifolds include almost all the closed manifolds which are known to have Riemannian metrics with nonnegative sectional curvature. (Examples are compact homogeneous Riemannian manifolds $G / H$, where $G$ is a compact Lie group, and more generally double coset manifolds $K \backslash G / H$ where $K$ is a subgroup of $G$ which acts freely on $G / H$.) Also, in all these cases, Burns's conjecture is correct; that is, the Lempert-Szóke-Guillemin-Stenzel complexification is an affine algebraic variety (Lemma 3.1).

Cheeger found the first simply connected closed manifolds of nonnegative sectional curvature which are not known to be double coset manifolds in 1973; his examples are the connected sum of any two rank-one symmetric spaces, with any orientations [5]. Remarkably, it took until the year 2000 for the next construction of closed manifolds of nonnegative curvature to be found, by Grove and Ziller; they showed that every manifold of cohomogeneity one for which the two singular orbits have codimension 2 has a metric of nonnegative curvature [11]. (Cohomogeneity one means that a compact Lie group acts on the manifold with a one-dimensional quotient space; the interesting case is where the quotient space is a closed interval, and the inverse images of the two endpoints are called the singular orbits.)

One main result of this paper is that all of Cheeger's manifolds and all closed manifolds of cohomogeneity one have good complexifications. In fact, Theorem 5.1 gives good complexifications for a certain class of manifolds which includes both of these types: roughly, unions of two disc bundles over manifolds with good complexifications. Thus all known closed manifolds with metrics of nonnegative curvature have good complexifications. We define these complexifications without using the Lempert-Szóke-Guillemin-Stenzel procedure, and it is natural to ask whether all manifolds of cohomogeneity one have metrics with entire Grauert tube. That is no doubt a hard problem: it would imply that all manifolds of cohomogeneity one have metrics of nonnegative curvature, as Grove and Ziller conjectured [11].

Finally, it is natural to try to classify the good complexifications of a given smooth manifold up to isomorphism. It is easy to see that $S^{1}$ has only one good complexification up to isomorphism. More interestingly, we show in Theorem 6.1 that $S^{2}$ and $\mathbf{R P}^{2}$ have only one good complexification if we strengthen the assumption that $U(\mathbf{R}) \rightarrow U(\mathbf{C})$ is a homotopy equivalence to say that $U(\mathbf{C})$ is diffeomorphic to the total space of the tangent bundle of $M=U(\mathbf{R})$, as is true in all known examples of good complexifications. The proof for $S^{2}$ and $\mathbf{R P}^{2}$ uses the methods of Ramanujam's topological characterization of $\mathbf{C}^{2}$ [21] and related results by Gurjar and Shastri [13]. Aguilar and Burns found the same result independently, with a similar proof [2].

In the course of classifying good complexifications of $S^{2}$ and $\mathbf{R} \mathbf{P}^{2}$, we show that the automorphism group of the real affine variety $S^{2}$ is the orthogonal group $O(3)$ (Theorem 6.2). The purpose of Sect. 7 is to show that the automorphism group of a good complexification can be noncompact or even infinite-dimensional. 
I am grateful to Róbert Szőke for allowing me to include in Sect. 7 his proof that the group of analytic automorphisms of $S_{\mathbf{C}}^{2}$ that commute with complex conjugation is infinite-dimensional. This answers a question in his paper [23].

\section{Topology of manifolds with good complexifications}

Kulkarni showed that a closed manifold $M$ which has a good complexification must have Euler characteristic $\chi(M) \geq 0$ [16]. We strengthen this result as follows. Our proof begins by repeating the proof of Kulkarni's theorem.

Theorem 1.1. Let $M$ be a connected closed manifold which has a good complexification. Then $\chi(M) \geq 0$. If $\chi(M)>0$, then all the odd Betti numbers of $M$ are 0 . If $\chi(M)=0$ and $M$ is oriented, then all the Pontrjagin numbers of $M$ are 0.

It is noteworthy that any rationally elliptic space has nonnegative Euler characteristic, and if the Euler characteristic is positive, then the odd Betti numbers are zero [7]. In view of Bott's conjecture that every manifold of nonnegative sectional curvature is rationally elliptic, we can view Theorem 1.1 as support for the possibility that every manifold with a good complexification has a metric of nonnegative sectional curvature.

Proof. If $M$ has odd dimension, then $\chi(M)=0$ and $M$ has no Pontrjagin numbers, these being defined only for a manifold of dimension a multiple of 4 . So there is only something to prove for $M$ of even dimension. Write $\operatorname{dim} M=2 k$. Also, if $M$ is not orientable, then we can replace $M$ by its orientable double cover, which again has a good complexification; so we can assume that $M$ is oriented.

We are given a good complexification of $M$, that is, a smooth affine variety $U$ over $\mathbf{R}$ such that $M$ is diffeomorphic to $U(\mathbf{R})$ and the inclusion $U(\mathbf{R}) \rightarrow U(\mathbf{C})$ is a homotopy equivalence. In particular, $U(\mathbf{C})$ is connected since $M$ is. (The proof of Theorem 1.1 works more generally if we assume only that $U$ is quasi-projective, as in Kulkarni [16], rather than affine. That generalization seems uninteresting, since all the motivating examples are affine varieties.)

By resolution of singularities, $U$ is an open dense subset of some smooth projective variety $X$ over $\mathbf{R}$ [14]. Let $D=X-U$. Since $U(\mathbf{R})$ is a closed manifold $M$, the set of real points of $X$ is the disjoint union of $M$ and the set of real points of $D$. But $X(\mathbf{R})$ is a $2 k$-dimensional manifold since $X$ is smooth, and $D(\mathbf{R})$ is a topological space of dimension at most $2 k-2$, so in fact $D(\mathbf{R})$ is empty and $X(\mathbf{R})=U(\mathbf{R})=M$.

The rational cohomology of any complex algebraic variety has a mixed Hodge structure. Since the inclusion $M \rightarrow U(\mathbf{C})$ is a homotopy equivalence, we get a mixed Hodge structure on $H^{*}(M, \mathbf{Q})$; since $U(\mathbf{C})$ is smooth, $H^{i}(M, \mathbf{Q})$ has weights between $i$ and $2 i$ for all $i$ [6].

Complex conjugation gives an action of the group $\mathbf{Z} / 2$ on $U(\mathbf{C})$, with $M$ as fixed point set. Since $\mathbf{Z} / 2$ acts trivially on $H^{*}(M, \mathbf{Q})$, it also acts trivially on $H^{*}(U(\mathbf{C}), \mathbf{Q})=H^{*}(M, \mathbf{Q})$. But for any variety such as $U$ which is defined over $\mathbf{R}$, 
complex conjugation acts on the mixed Hodge structure of $H^{*}(U(\mathbf{C}), \mathbf{Q})$ in such a way that, if we write the Hodge decomposition of the weight-graded pieces as

$$
g r_{a}^{W} H^{b}(U(\mathbf{C}), \mathbf{C})=\oplus_{j} V^{j, a-j}
$$

then conjugation maps $V^{j, a-j}$ to $V^{a-j, j}$. Since conjugation acts trivially on $H^{*}(U(\mathbf{C}), \mathbf{Q})$, it follows that

$$
g r_{a}^{W} H^{b}(U(\mathbf{C}), \mathbf{C})=V^{a / 2, a / 2}
$$

for all $a$ and $b$. That is, the weight filtration on each cohomology group of $U(\mathbf{C})$, or equivalently of $M$, is concentrated in even weights, and the Hodge structure on each weight-graded piece is trivial.

In particular, let $x \in H^{2 k}(X(\mathbf{C}), \mathbf{Q})$ be the cohomology class represented by $M$ in $X$, using the given orientation of $M$. Then $x$ is nonzero if and only if the homomorphism from $\mathbf{Q}=H_{2 k}(M, \mathbf{Q})$ to $H_{2 k}(X(\mathbf{C}), \mathbf{Q})$ is not zero, thus if and only if the dual homomorphism from $H^{2 k}(X(\mathbf{C}), \mathbf{Q})$ to $H^{2 k}(U(\mathbf{C}), \mathbf{Q})=$ $H^{2 k}(M, \mathbf{Q})=\mathbf{Q}$ is not zero. By definition of the weight filtration, this means that $x$ is nonzero if and only if $H^{2 k}(M, \mathbf{Q}) \cong \mathbf{Q}$ is in weight $2 k$ (a priori, it could be anywhere from weight $2 k$ to weight $4 k$ ). Moreover, the previous paragraph shows that if $x$ is nonzero, then it is in the $(k, k)$ subspace of the Hodge decomposition of $H^{2 k}(X(\mathbf{C}), \mathbf{C})$.

The final observation needed to prove Kulkarni's theorem is that the class $x \in H^{2 k}(X(\mathbf{C}), \mathbf{Q})$ is primitive, meaning that $x L=0 \in H^{2 k+2}(X(\mathbf{C}), \mathbf{Q})$ where $L \in H^{2}(X(\mathbf{C}), \mathbf{Q})$ is the class of an ample line bundle on $X$. This is a general property of the cohomology class represented by the real locus in a complex projective variety defined over $\mathbf{R}$. Namely, one uses some multiple of $L$ to give a projective embedding $X \rightarrow \mathbf{P}^{N}$ defined over $\mathbf{R}$, so that $X(\mathbf{R})=M$ maps into $\mathbf{P}^{N}(\mathbf{R})$; then the divisor $x_{0}^{2}+\cdots+x_{N}^{2}=0$ is ample on $P^{N}$ and hence pulls back to an ample divisor on $X$, but its intersection with the real locus is empty. It follows that $x L=0 \in H^{2 k+2}(X(\mathbf{C}), \mathbf{Q})$.

The normal bundle of $M \subset X(\mathbf{C})$ is isomorphic to the tangent bundle of $M$ as a real vector bundle, as is true for any real algebraic variety. So the self-intersection number $x^{2} \in H^{4 k}(X(\mathbf{C}), \mathbf{Q})=\mathbf{Q}$ is, up to a sign, the Euler class of $T M$ in $H^{2 k}(M, \mathbf{Q})=\mathbf{Q}$, that is, the Euler characteristic of $M$. To be precise ([16], p. 61):

$$
x^{2}=(-1)^{k} \chi(M) \text {. }
$$

Thus, if $x=0 \in H^{2 k}(X(\mathbf{C}), \mathbf{Q})$, then $M$ has Euler characteristic 0 . If $x$ is not 0 , then it is a nonzero primitive cohomology class in the $(k, k)$ subspace of $H^{2 k}(X(\mathbf{C}), \mathbf{C})$, and so the Hodge-Riemann bilinear relations ([9], p. 123) give that

$$
(-1)^{k} x^{2}>0 \text {. }
$$

Thus $\chi(M)>0$ if $x \neq 0$. This concludes Kulkarni's proof that $\chi(M) \geq 0$.

We now prove the more precise statements in the theorem. First, suppose that $\chi(M)>0$. By the above proof, this means that the class $x$ of $M$ in the rational cohomology of $X(\mathbf{C})$ is not zero, or equivalently that the mixed Hodge structure on $H^{*}(M, \mathbf{Q})$ which comes from its identification with $H^{*}(U(\mathbf{C}), \mathbf{Q})$ 
has $H^{2 k}(M, \mathbf{Q})=\mathbf{Q}$ in weight $2 k$. Recall that $H^{i}(M, \mathbf{Q})$ is in weights $\geq i$ for all $i$. Moreover, the rational cohomology ring of $M$ is isomorphic to the associated graded ring with respect to the weight filtration [6]. In this associated graded ring, the product of $g r_{a}^{W} H^{b}(M, \mathbf{Q})$ for any $a>b$ with all of $H^{2 k-b}(M, \mathbf{Q})$ is 0 , because it lands in $g r_{c}^{W} H^{2 k}(M, \mathbf{Q})$ for $c>2 k$ which is 0 . Since $H^{*}(M, \mathbf{Q})$ satisfies Poincaré duality, it follows that $H^{i}(M, \mathbf{Q})$ is pure of weight $i$ for all $i$. Since we showed earlier that $H^{*}(M, \mathbf{Q})$ is a mixed Hodge structure with all weights even, it follows that $H^{i}(M, \mathbf{Q})=0$ for $i$ odd, as we want.

We now prove the final statement of the theorem. We suppose that $\chi(M)=0$, and we will show that the Pontrjagin numbers of $M$ are 0 . We can assume that $M$ has dimension of the form $4 a$; otherwise there are no Pontrjagin numbers. Since $\chi(M)=0$, the above proof shows that the rational cohomology class represented by $M$ in $X(\mathbf{C})$ is 0 ; equivalently, the mixed Hodge structure on $H^{4 a}(M, \mathbf{Q})=\mathbf{Q}$ is in some weight greater than $4 a$.

On the other hand, as for any real algebraic variety, the tangent bundle of $X(\mathbf{C})$ restricted to $M$ is $T M \otimes_{\mathbf{R}} \mathbf{C}$. So the Pontrjagin classes of $M$,

$$
p_{i}(M)=(-1)^{i} c_{2 i}\left(T M \otimes_{\mathbf{R}} \mathbf{C}\right) \in H^{4 i}(M, \mathbf{Q}),
$$

are, up to sign, the pullbacks to $M$ of the even Chern classes of $T X$ on $X(\mathbf{C})$. The subring of $H^{*}(M, \mathbf{Q})$ of lowest weight (weight $i$ in $H^{i}$ ) is the image of restriction from $H^{*}(X(\mathbf{C}), \mathbf{Q})$, by the definition of the mixed Hodge structure on $H^{*}(M, \mathbf{Q})=H^{*}(U(\mathbf{C}), \mathbf{Q})$. So the Pontrjagin classes of $M$ lie in this lowestweight subring of $H^{*}(M, \mathbf{Q})$. Since the top-degree cohomology of $M, H^{4 a}(M, \mathbf{Q})$ is in some weight greater than $4 a$, any product of Pontrjagin classes in the topdegree cohomology of $M$ is 0 . That is, the Pontrjagin numbers of $M$ are 0 .

\section{Stronger restrictions on manifolds with good complexifications}

The proof of Theorem 1.1 implies more restrictions on which manifolds have good complexifications than stated there. We formulate a stronger but more elaborate statement as follows, the proof being the same as for Theorem 1.1. This stronger statement, unlike Theorem 1.1, gives some restrictions on which odd-dimensional manifolds can have good complexifications, as we will discuss below.

Theorem 2.1. Let $M$ be a closed n-manifold which has a good complexification. Then there is a grading on the rational homotopy type of $M$ called the weight grading. This makes $H^{*}(M, \mathbf{Q})$ into a bigraded ring, with the following properties:

(1) Each group $H^{i}(M, \mathbf{Q})$ has weights between $i$ and $2 i$, and all weights are even. Moreover, for M orientable, $H^{*}(M, \mathbf{Q})$ satisfies Poincaré duality as a bigraded ring.

(2) If $\chi(M)>0$, then $H^{i}(M, \mathbf{Q})$ is in weight $i$ for all $i$, and is 0 for $i$ odd. If $\chi(M)=0$, then the top-degree group $H^{n}(M, \mathbf{Q})(\cong \mathbf{Q}$, if $M$ is orientable $)$ is in some weight greater than $n$.

(3) The Pontrjagin classes of $M$ are in the bottom-weight subring, meaning that $p_{i}(M)$ is in the weight $4 i$ summand of $H^{4 i}(M, \mathbf{Q})$. 
For example, $M=S^{1} \times S^{2}$ has a good complexification with weights on $H^{*}(M, \mathbf{Q})$ as follows:

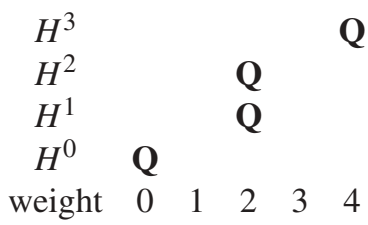

As promised, the theorem says something for odd-dimensional manifolds as well as for even-dimensional manifolds. For example, if $M$ is an orientable 3-manifold with a good complexification, then $H^{3}(M, \mathbf{Q}) \cong \mathbf{Q}$ must be in weight either 4 or 6 . In the first case, $H^{1}(M, \mathbf{Q})$ is in weight 2 and, by Poincare duality, $H^{2}(M, \mathbf{Q})$ is in weight 2. It follows that the product

$$
H^{1}(M, \mathbf{Q}) \otimes H^{1}(M, \mathbf{Q}) \rightarrow H^{2}(M, \mathbf{Q})
$$

is identically 0 . In the second case $\left(H^{3}(M, \mathbf{Q})\right.$ in weight 6$)$, Poincaré duality shows that $H^{i}(M, \mathbf{Q})$ is in weight $2 i$ for all $i$. It follows that the rational homotopy type of $M$ is formal. Thus, if $M$ is an orientable 3-manifold with a good complexification, then either the product map $H^{1} \otimes H^{1} \rightarrow H^{2}$ is 0 with rational coefficients or else $M$ is formal. One can find other restrictions on which 3-manifolds have good complexifications using the known restrictions on the possible fundamental groups of smooth complex algebraic varieties.

Be careful that the existence of a grading on the rational homotopy type of $M$ as in the theorem does not say by itself that $M$ is formal. We only see that $M$ is formal if there is a constant $k$ such that $H^{i}(M, \mathbf{Q})$ is in weight $k i$ for all $k$. For example, there are homogeneous spaces $G / H$ for compact Lie groups which are not formal ([8], Chap. 11, Sect. 5). Since all homogeneous spaces have good complexifications, the properties in the theorem do not imply formality in general. On the other hand, we do have formality if we can control the weights of $M$ in some way. For example, if $M$ is a manifold with good complexification which has positive Euler characteristic (such as a homogeneous space with positive Euler characteristic), then the theorem says that $H^{i}(M, \mathbf{Q})$ is in weight $i$ for all $i$, and so $M$ is formal.

The known examples of manifolds $M$ with good complexifications, which are roughly homogeneous spaces and some related manifolds (see Sects. 3, 4, and 5), suggest that the weight grading on the homotopy type of a manifold with good complexification should be essentially determined by the homotopy type. Precisely, say assuming that $M$ is a nilpotent space, the grading should have all of $\pi_{i}(M) \otimes \mathbf{Q}$ in weight $-i$ if $i$ is even, and weight $-(i+1)$ if $i$ is odd; Y. Félix called rationally elliptic spaces with such a weight grading "pure elliptic spaces" [7]. For example, this conjecture would say that any good complexification of $S^{n}$ has $H^{n}\left(S^{n}, \mathbf{Q}\right)$ in weight $n$ if $n$ is even and in weight $n+1$ if $n$ is odd, as is true for the standard complexification of $S^{n}$. Also, this conjecture would imply that it is very rare to have a good complexification of an orientable $n$-manifold $M$ such that $H^{n}(M, \mathbf{Q}) \cong \mathbf{Q}$ is in the highest possible weight, $2 n$ : in fact, the only examples I know of such a manifold are the flat Riemannian manifolds, and we can guess that they are the only manifolds of this type. 


\section{Good complexifications of quotient manifolds}

In this section we show that for a compact Lie group $G$ acting on a closed manifold $M$, if $M$ has a $G$-equivariant good complexification, then the quotient manifold $M / G$ has a good complexification. This is completely analogous to the known results that (1) the quotient of a Riemannian manifold of nonnegative sectional curvature by a Lie group acting freely by isometries also has nonnegative sectional curvature, by O'Neill's curvature formula [5], and (2) the quotient of a Riemannian manifold with entire Grauert tube by a Lie group acting freely by isometries also has entire Grauert tube, by Aguilar [1].

In particular, for a compact Lie group $G$, homogeneous spaces $G / H$ and double coset manifolds $K \backslash G / H$ (where $K$ acts freely on $G / H$ ) have good complexifications. Also, in Sect. 5 we will show that manifolds with a $G$-action of cohomogeneity one have $G$-equivariant good complexifications, whereas they are not yet known to have metrics with entire Grauert tube. Then we can use Lemma 3.1 to show that quotients of such a manifold by a subgroup of $G$ which acts freely also have good complexifications.

Lemma 3.1. Let $U$ be a good complexification with an action of the complexification $G$ of a compact Lie group $G(\mathbf{R})$. If $G(\mathbf{R})$ acts freely on $U(\mathbf{R})$, then the algebraic group $G$ acts freely on $U$, and the quotient variety $U / G$ is a good complexification of the quotient manifold $U(\mathbf{R}) / G(\mathbf{R})$.

Proof. One of the main results of the Smith theory of group actions is as follows. Let $X$ and $Y$ be finite-dimensional topological spaces with actions of the group $\mathbf{Z} / p$. Suppose we have an equivariant map $X \rightarrow Y$ which is an isomorphism on cohomology with $\mathbf{Z} / p$ coefficients. Then the map of fixed point sets $X^{\mathbf{Z} / p} \rightarrow Y^{\mathbf{Z} / p}$ is also an isomorphism on cohomology with $\mathbf{Z} / p$ coefficients ([3], p. 145). In particular, if $\mathbf{Z} / p$ acts freely on $X$, then it acts freely on $Y$.

We apply this fact to the inclusion $U(\mathbf{R}) \rightarrow U(\mathbf{C})$, which is a homotopy equivalence and also an equivariant map for the action of $G(\mathbf{R})$. Any nontrivial stabilizer subgroup for the compact Lie group $G(\mathbf{R})$ acting on $U(\mathbf{C})$ must contain the group $\mathbf{Z} / p$ for some prime $p$. Since $G(\mathbf{R})$ acts freely on $U(\mathbf{R})$, Smith theory as explained above shows that $G(\mathbf{R})$ also acts freely on $U(\mathbf{C})$. Also, we are assuming that $G$ is the complexification of the compact Lie group $G(\mathbf{R})$, which implies that $G(\mathbf{R})$ is a maximal compact subgroup of $G(\mathbf{C})$. So any compact subgroup of $G(\mathbf{C})$ is conjugate to a subgroup of $G(\mathbf{R})$ and hence acts freely on $U(\mathbf{C})$.

Suppose that $G$ has a closed orbit in $U$ with nontrivial stabilizer group $H$. Then the orbit $G / H$ is affine since $U$ is affine, so the subgroup $H$ is reductive by Matsushima [18]. Then $H(\mathbf{C})$ contains a nontrivial compact subgroup, contradicting the fact that every compact subgroup of $G(\mathbf{C})$ acts freely on $U(\mathbf{C})$. So in fact every closed orbit of $G$ on $U$ has trivial stabilizer.

If $G$ has a non-closed orbit in $U$, then the closure of this orbit contains a lowerdimensional closed orbit, contrary to what we have shown. So the orbits are all closed and have trivial stabilizer. Since $U$ is affine and $G$ is reductive, geometric invariant theory now shows that the action of $G$ on $U$ is free (in particular, proper) 
and also that there is a geometric quotient variety $U / G$, which is a smooth affine variety over $\mathbf{R}$ ([20], p. 30 and p. 16).

Since $G$ acts freely on $U$, the orbit of a point of $U(\mathbf{R})$ is isomorphic to $G$ as a variety over $\mathbf{R}$, which implies that the topological quotient $U(\mathbf{R}) / G(\mathbf{R})$ maps injectively to $(U / G)(\mathbf{R})$. Thus we know that one connected component of the manifold of real points of $U / G$ is equal to the closed manifold $U(\mathbf{R}) / G(\mathbf{R})$. This one connected component of the real points is homotopy equivalent to the complex points of $U / G$. That there are no other connected components of the space of real points follows from Smith's inequalities, which give that the sum of the $\mathbf{Z} / 2$-Betti numbers of the real points of any real algebraic variety is at most the sum of the $\mathbf{Z} / 2$-Betti numbers of the complex points ([3], p. 144). Lemma 3.1 is proved.

\section{Good complexifications for Cheeger's manifolds}

In this section we construct good complexifications for a class of manifolds which includes all of Cheeger's manifolds. By definition, Cheeger's manifolds are the connected sums of two rank-one symmetric spaces, with any orientations. The next section will in fact construct good complexifications for a broader class of manifolds, but the proof will be modeled on the simpler proof in this section.

Definition. Let $E$ be a good complexification, that is, a smooth affine variety over $\mathbf{R}$ such that $E(\mathbf{R})$ is compact and the map $E(\mathbf{R}) \rightarrow E(\mathbf{C})$ is a homotopy equivalence. We say that $E$ is a sphere bundle over another variety $B$ if there is a good complexification $P_{B}$ with a free action of the orthogonal group $O(b)$ of the quadratic form $x_{0}^{2}+\cdots+x_{b-1}^{2}$ such that $E=P_{B} / O(b-1)$ and $B=P_{B} / O(b)$. In this case, we have a fibration $S^{b-1} \rightarrow E \rightarrow B$, where $S^{b-1}$ here means the real affine variety defined by $x_{0}^{2}+\cdots+x_{b-1}^{2}=1$. (Sometimes we write $S^{b-1}$ for the manifold of real points $S^{b-1}(\mathbf{R})$, as usual; the context should make it clear what is meant.)

Theorem 4.1. Let $E$ be a good complexification which is a sphere bundle in two ways, $S^{b-1} \rightarrow E \rightarrow B$ and $S^{c-1} \rightarrow E \rightarrow C$. Suppose that the two Z/2-actions on $E$ given by the antipodal maps in the two sphere bundles are the same.

Let $M$ be the closed manifold which is the union of the two disc bundles associated to the sphere bundles $S^{b-1} \rightarrow E(\mathbf{R}) \rightarrow B(\mathbf{R})$ and $S^{c-1} \rightarrow E(\mathbf{R}) \rightarrow$ $C(\mathbf{R})$ along their common boundary, $E(\mathbf{R})$. Then $M$ has a good complexification.

Corollary 4.2. Cheeger's manifolds, the connected sums of any two rank-one symmetric spaces of the same dimension $\left(\mathbf{R} \mathbf{P}^{n}, \mathbf{C} \mathbf{P}^{n}, \mathbf{H} \mathbf{P}^{n}\right.$, or $\left.C a \mathbf{P}^{2}\right)$ with any orientations, have good complexifications.

Proof of Corollary 4.2. A Cheeger manifold $M$ is the union of two disc bundles meeting in their common boundary $N$, which is the sphere of some dimension. The two sphere bundles with total space $N$ both have the form $S^{0} \rightarrow S^{n-1} \rightarrow \mathbf{R P}^{n-1}$, $S^{1} \rightarrow S^{2 n-1} \rightarrow \mathbf{C P}^{n-1}, S^{3} \rightarrow S^{4 n-1} \rightarrow \mathbf{H P}^{n-1}$, or $S^{7} \rightarrow S^{15} \rightarrow S^{8}$. In all these cases, the corresponding principal $O(b)$-bundles have explicit descriptions 
as quotients of homogeneous spaces, so that they have $O(b)$-equivariant good complexifications by Lemma 3.1:

$$
\begin{array}{r}
O(1) \rightarrow S^{n-1} \rightarrow \mathbf{R P}^{n-1} \\
O(2) \rightarrow\left(S^{2 n-1} \times O(2)\right) / S^{1} \rightarrow \mathbf{C P}^{n-1} \\
O(4) \rightarrow\left(S^{4 n-1} \times O(4)\right) / S^{3} \rightarrow \mathbf{H P}^{n-1} \\
O(8) \rightarrow(\operatorname{Spin}(9) \times O(8)) / \operatorname{Spin}(8) \rightarrow S^{8} .
\end{array}
$$

In the last case, $\operatorname{Spin}(8) \rightarrow \operatorname{Spin}(9)$ is the standard inclusion, with $\operatorname{Spin}(9) / \operatorname{Spin}(8)$ $=S^{8}$, and $\operatorname{Spin}(8) \rightarrow O(8)$ is one of the two spin representations.

Moreover, in all these cases, the antipodal map on each fiber of the sphere bundle gives the antipodal map on the total space $N$, viewed as a sphere. It follows that the antipodal maps for the two sphere bundles with total space $N$ are the same; in particular, they both preserve the standard metric on the sphere $N$. This is true whether our choice of identification between the total spaces of the two sphere bundles preserves orientation or not. Then Theorem 4.1 shows that the union of the two corresponding disc bundles has a good complexification, as we want.

Proof of Theorem 4.1. As a first step, define a manifold $M_{B}$ as the union of two copies of the same disc bundle, the one associated to the sphere bundle $S^{b-1} \rightarrow$ $E(\mathbf{R}) \rightarrow B(\mathbf{R})$, joined along their common boundary. We will construct a good complexification of $M_{B}$. We are given that this sphere bundle has structure group $O(b)$, so that there is a corresponding principal bundle $O(b) \rightarrow P_{B}(\mathbf{R}) \rightarrow B(\mathbf{R})$. Using the standard inclusion $O(b) \subset O(b+1)$, the group $O(b)$ acts on the sphere $S^{b}$, fixing two antipodal points. Then $M_{B}$ can be identified with $\left(P_{B}(\mathbf{R}) \times S^{b}\right) / O(b)$. This construction shows that $M_{B}$ is a fiber bundle over $B(\mathbf{R})$ with fiber $S^{b}$. Since we are given that $P_{B}(\mathbf{R})$ has an $O(b)$-equivariant good complexification, and the homogeneous space $S^{b}$ has an $O(b)$-equivariant (even $O(b+1)$-invariant) good complexification, their product also has an $O(b)$-equivariant good complexification. By Lemma 3.1, the quotient manifold $M_{B}$ has a good complexification $U_{B}$, as we want.

We need to look more closely at the real algebraic variety $U_{B}:=\left(P_{B} \times\right.$ $\left.S^{b}\right) / O(b)$ produced by this construction. There is an algebraic map

$$
f: U_{B} \rightarrow A_{\mathbf{R}}^{1}
$$

defined by projecting to $S^{b} / O(b)$ and identifying this geometric quotient with $A_{\mathbf{R}}^{1}$. This last identification is just the projection map from $S^{b} \subset A_{\mathbf{R}}^{b+1}$ to $A_{\mathbf{R}}^{1}$ using the last coordinate, $x_{b}$. Let $U_{B}^{0}$ be the inverse image of $A^{1}-\{ \pm 1\}$ in the variety $U_{B}$.

Lemma 4.3. There is an isomorphism of real algebraic varieties

$$
U_{B}^{0} \cong\left(E \times\left(S^{1}-\{ \pm 1\}\right)\right) / \mathbf{Z} / 2 .
$$

Here $\mathbf{Z} / 2$ acts on the circle $S^{1}$ by the reflection $z \mapsto z^{-1}$, and on $E$ by the antipodal map associated to the sphere bundle $S^{b-1} \rightarrow E \rightarrow B$. 
That is, a certain explicit Zariski open subset of $U_{B}$ does not depend on the sphere bundle $S^{b-1} \rightarrow E \rightarrow B$, but only on the antipodal map on $E$ associated to this sphere bundle.

Proof. The point is that the real algebraic projection map

$$
x_{b}: S^{b} \rightarrow A_{\mathbf{R}}^{1}
$$

over the open subset $A^{1}-\{ \pm 1\}$, is a fiber bundle with fiber $S^{b-1}$ and structure group $\mathbf{Z} / 2$. (If we only look at real points, we do not notice that structure group; the map just looks like a trivial fiber bundle over the open interval $(-1,1)$.) More explicitly, there is a real algebraic isomorphism

$$
\left(S^{b-1} \times\left(S^{1}-\{ \pm 1\}\right)\right) / \mathbf{Z} / 2 \cong S^{b}-\left\{x_{b}= \pm 1\right\},
$$

where $\mathbf{Z} / 2$ acts on $S^{b-1}$ by the antipodal map and on $S^{1}$ by the reflection $z \mapsto z^{-1}$, or equivalently $(x, y) \mapsto(x,-y)$. The isomorphism is defined by

$$
\left(\left(x_{0}, \ldots, x_{b-1}\right),(x, y)\right) \mapsto\left(x_{0} y, \ldots, x_{b-1} y, x\right) .
$$

It follows that the inverse image $U_{B}^{0}$ of $A^{1}-\{ \pm 1\}$ in $U_{B}$ satisfies

$$
\begin{aligned}
U_{B}^{0} & \cong\left(P_{B} \times\left(S^{b}-\left\{x_{b}= \pm 1\right\}\right)\right) / O(b) \\
& \cong\left(P_{B} \times S^{b-1} \times\left(S^{1}-\{ \pm 1\}\right)\right) /(O(b) \times \mathbf{Z} / 2) .
\end{aligned}
$$

Here $O(b)$ acts on $P_{B}$ and on $S^{b-1}$, while $\mathbf{Z} / 2$ acts on $S^{b-1}$ by the antipodal map and on $S^{1}$ by the reflection $z \mapsto z^{-1}$. By viewing $S^{b-1}$ as $O(b) / O(b-1)$, we see that

$$
U_{B}^{0} \cong\left(P_{B} \times\left(S^{1}-\{ \pm 1\}\right)\right) /(O(b-1) \times \mathbf{Z} / 2),
$$

where $O(b-1)$ acts on $P_{B}$ and $\mathbf{Z} / 2$ now acts on $P_{B}$ by -1 in $O(b)$, as well as acting on $S^{1}$ as before. Since $E=P_{B} / O(b-1)$, we have

$$
U_{B}^{0} \cong\left(E \times\left(S^{1}-\{ \pm 1\}\right)\right) / \mathbf{Z} / 2
$$

as we want, with $\mathbf{Z} / 2$ acting on $E$ by the antipodal map on the fibers of the sphere bundle $S^{b-1} \rightarrow E \rightarrow B$, and on $S^{1}$ as before.

We can now construct a good complexification of $M$, the union of two disc bundles, using that the two $\mathbf{Z} / 2$-actions on $E$ are the same. We have constructed a real algebraic variety $U_{B}$ associated to the fibration $S^{b-1} \rightarrow E \rightarrow B$. We can apply the same construction to define another real algebraic variety $U_{C}$ associated to the fibration $S^{c-1} \rightarrow E \rightarrow C$. Both varieties come with maps to the affine line $A_{\mathbf{R}}^{1}$. Lemma 4.3 gives an explicit isomorphism from the inverse image of $A^{1}-\{ \pm 1\}$ in $U_{B}$ to the inverse image of $A^{1}-\{ \pm 1\}$ in $U_{C}$, because the two $\mathbf{Z} / 2$-actions on $E$ are the same.

Therefore, we can define a real algebraic variety $U$ by pasting together the inverse image of $A^{1}-\{1\}$ in $U_{B}$ and the inverse image of $A^{1}-\{-1\}$ in $U_{C}$ along 
their common open subset, the inverse image of $A^{1}-\{ \pm 1\}$. By construction, we have a morphism

$$
f: U \rightarrow A_{\mathbf{R}}^{1}
$$

The inverse images of $A^{1}-\{1\}$ and $A^{1}-\{-1\}$ are both affine varieties, and so $f$ is an affine morphism. Since $A^{1}$ is also affine, it follows that $U$ is an affine variety. It is smooth since $U_{B}$ and $U_{C}$ are.

Clearly $U(\mathbf{R})$ is diffeomorphic to the given manifold $M$ : we have a proper map $f$ from $U(\mathbf{R})$ onto the interval $[-1,1]$, which is a fibration over $(-1,1)$ with fiber $E(\mathbf{R})$, and which has fiber $B(\mathbf{R})$ over -1 and $C(\mathbf{R})$ over 1 .

It remains to show that the inclusion $U(\mathbf{R}) \rightarrow U(\mathbf{C})$ is a homotopy equivalence. We will use the map $f: U(\mathbf{C}) \rightarrow \mathbf{C}$. Define open subsets $S, T \subset \mathbf{C}$ by

$$
\begin{aligned}
& S=\{z \in \mathbf{C}: \operatorname{Re} z<1 / 2\} \\
& T=\{z \in \mathbf{C}: \operatorname{Re} z>-1 / 2\} .
\end{aligned}
$$

Clearly $S \cup T=\mathbf{C}$. Therefore, to show that $U(\mathbf{R}) \rightarrow U(\mathbf{C})$ is a homotopy equivalence, it suffices to show that $f^{-1}(S)(\mathbf{R}) \rightarrow f^{-1}(S)(\mathbf{C}), f^{-1}(T)(\mathbf{R}) \rightarrow$ $f^{-1}(T)(\mathbf{C})$, and $f^{-1}(S \cap T)(\mathbf{R}) \rightarrow f^{-1}(S \cap T)(\mathbf{C})$ are homotopy equivalences.

We use that $f: U(\mathbf{C}) \rightarrow \mathbf{C}$ is topologically a fibration outside \pm 1 , with fiber $E(\mathbf{C})$, while $f: U(\mathbf{R}) \rightarrow[-1,1]$ is a fibration outside \pm 1 with fiber $E(\mathbf{R})$. Since $S \cap T$ is contractible and does not contain \pm 1 , the inverse image of $S \cap T$ in $U(\mathbf{C})$ is homotopy equivalent to a single fiber $E(\mathbf{C})$. Likewise, $S \cap T \cap[-1,1]$ is contractible and does not contain \pm 1 , so its inverse image in $U(\mathbf{R})$ is homotopy equivalent to a single fiber $E(\mathbf{R})$. We know that $E$ is a good complexification, so that $E(\mathbf{R}) \rightarrow E(\mathbf{C})$ is a homotopy equivalence. It follows that $f^{-1}(S \cap T)(\mathbf{R}) \rightarrow$ $f^{-1}(S \cap T)(\mathbf{C})$ is a homotopy equivalence.

Since the map $f: U(\mathbf{R}) \rightarrow[-1,1]$ is proper and a fibration outside \pm 1 , the inverse image of $S$ in $U(\mathbf{R})$ is homotopy equivalent to the inverse image of the point -1 , which is the manifold $B(\mathbf{R})$. Likewise, the inverse image of $S$ in $U(\mathbf{C})$ is homotopy equivalent to the inverse image of a small ball around -1 ; since the map $f: U(\mathbf{C}) \rightarrow \mathbf{C}$ is not proper, it is not immediate that this inverse image is homotopy equivalent to the inverse image of the single point -1 . We need the following lemma.

Lemma 4.4. The inverse image of a small ball around -1 under the map $x_{b}$ : $S^{b}(\mathbf{C}) \rightarrow \mathbf{C}$ is contractible.

Proof. Projectivizing the coordinates in $A^{b+1}$ other than $x_{b}$ exhibits the affine variety $S^{b}(\mathbf{C})$ as the complement of a divisor $Z$ in a closed hypersurface $Y$ in $\mathbf{P}^{b} \times A^{1}$, so that $Y$ is proper over $A^{1}$. Here $Z$ is smooth over $A^{1}$ and the proper map $Y \rightarrow A^{1}$ is smooth except at the two points $\left(x_{0}, \ldots, x_{b-1}, x_{b}\right)=(0, \ldots, 0, \pm 1)$ in the open subset $S^{b}(\mathbf{C})$ of $Y$. This is enough to ensure that the inverse image of a small ball around -1 in $S^{b}(\mathbf{C})$ is homotopy equivalent to the inverse image of the point -1 . Furthermore, the latter inverse image is an affine quadric cone, and therefore contractible. 
We have said that $f^{-1}(S)(\mathbf{C})$ is homotopy equivalent to the inverse image of a small ball around -1 in $U(\mathbf{C})$. By the construction of $U$, this is isomorphic to the inverse image of a small ball around -1 in $U_{B}(\mathbf{C})=\left(P_{B}(\mathbf{C}) \times S^{b}(\mathbf{C})\right) / O(b, \mathbf{C})$. By Lemma 4.4, this is homotopy equivalent to $P_{B}(\mathbf{C}) / O(b, \mathbf{C})=B(\mathbf{C})$. We know that $B(\mathbf{R}) \rightarrow B(\mathbf{C})$ is a homotopy equivalence since $B$ is a good complexification. So we have shown that $f^{-1}(S)(\mathbf{R}) \rightarrow f^{-1}(S)(\mathbf{C})$ is a homotopy equivalence. The same argument works for $T$, using that $C$ is a good complexification. It follows that $U(\mathbf{R}) \rightarrow U(\mathbf{C})$ is a homotopy equivalence. Thus $U$ is a good complexification of the given manifold $M$.

$\square($ Theorem 4.1)

\section{Good complexifications for manifolds of cohomogeneity one and related manifolds}

In this section we construct good complexifications for a class of manifolds which includes both Cheeger's manifolds (already treated in Sect. 4) and manifolds with a cohomogeneity-one action of a compact Lie group. The interest of these two classes of manifolds is that the only known closed manifolds which have Riemannian metrics of nonnegative curvature are obtained by taking products and forming quotients by groups of isometries, starting with the real line, compact Lie groups, Cheeger's manifolds [5], and Grove and Ziller's manifolds, that is, manifolds with a cohomogeneity-one action of a compact Lie group such that the singular orbits have codimension 2 [11].

We will take care to find a $G$-equivariant good complexification for each manifold with a cohomogeneity-one action of a compact Lie group $G$, for the following reason. Grove and Ziller showed that 10 of the 14 diffeomorphism classes of exotic 7-spheres are quotients of cohomogeneity-one manifolds $M$ by a subgroup of $G$ which acts freely on $M$ [11]. Thus Corollary 5.2 together with Lemma 3.1 shows that these same 10 of the 14 diffeomorphism classes of exotic 7-spheres admit good complexifications.

For those exotic spheres, Grove and Ziller found metrics of nonnegative sectional curvature. There are other exotic spheres, namely the Kervaire spheres (of dimension $9,13, \ldots$ ), which admit cohomogeneity-one group actions by Hsiang and Hsiang [15]. So they have good complexifications by Corollary 5.2. It has been conjectured for 30 years that the Kervaire spheres should have metrics of nonnegative sectional curvature [5], but that is still unknown.

We will actually construct good complexifications for a class of manifolds which includes both Cheeger's manifolds and manifolds of cohomogeneity one with quotient space equal to the closed interval, as follows. Recall the definition of when a good complexification is a sphere bundle, before Theorem 4.1.

Theorem 5.1. Let $E$ be a good complexification which is a sphere bundle in two ways, $S^{b-1} \rightarrow E \rightarrow B$ and $S^{c-1} \rightarrow E \rightarrow C$. Suppose that the closed manifold $E(\mathbf{R})$ has a Riemannian metric which is preserved by the two $\mathbf{Z} / 2$-actions on $E(\mathbf{R})$ given by the antipodal maps in the two sphere bundles.

Let $M$ be the closed manifold which is the union of the two disc bundles associated to the sphere bundles $S^{b-1} \rightarrow E(\mathbf{R}) \rightarrow B(\mathbf{R})$ and $S^{c-1} \rightarrow E(\mathbf{R}) \rightarrow C(\mathbf{R})$ 
along their common boundary, $E(\mathbf{R})$. Then $M$ has a good complexification $U$. If we have a compact Lie group $K$ which acts algebraically on $E$, compatibly on $E$, $B$, and $C$, preserving the standard metrics on the spheres $S^{b-1}$ and $S^{c-1}$ as well as the above metric on $E(\mathbf{R})$, then $K$ acts on $M$ and the complexification $G$ of $K$ acts on $U$.

Corollary 5.2. Any closed manifold $M$ with a cohomogeneity-one action of a compact Lie group $K$ has a good complexification with an action of the complexification $G$ of $K$.

Proof of Corollary 5.2. The quotient space $M / K$ is homeomorphic either to the circle or to a closed interval [19]. In the first case, $M$ is a bundle over the circle with fiber a homogeneous space $K / H$. More precisely, $M$ is the bundle associated to some $K$-map from $K / H$ to itself. Such a map has the form $x H \mapsto x a H$ for a uniquely determined element $a \in N_{K}(H) / H$. Here $M$ only depends on the connected component of the group $N_{K}(H) / H$ which contains $a$, so we can assume that $a$ is a torsion element of $N_{K}(H) / H$, say $a^{n}=1$. Then $M$ is the quotient manifold $\left(K / H \times S^{1}\right) /(\mathbf{Z} / n)$. So $M$ has a good complexification with an action of the complexification $G$ of $K$, by Lemma 3.1.

It remains to consider the more complicated manifolds of cohomogeneity one, those with $M / K$ homeomorphic to a closed interval. In this case, the general fiber of $M$ has the form $K / A$ for some closed subgroup $A$, and the fibers over the endpoints have the form $K / B$ and $K / C$ for some subgroups $B$ and $C$ containing $A$. Moreover, $B / A$ and $C / A$ are both diffeomorphic to spheres, $S^{b-1}$ and $S^{c-1}$, with $B$ and $C$ acting by isometries in the usual metric on the sphere [11]. Finally, the whole manifold $M$ is the union of the two disc bundles associated to the sphere bundles $S^{b-1} \rightarrow K / A \rightarrow K / B$ and $S^{c-1} \rightarrow K / A \rightarrow K / C$, identified along their common boundary $K / A$.

Since $B$ and $C$ act by isometries on $S^{b-1}$ and $S^{c-1}$, we have homomorphisms $B \rightarrow O(b)$ and $C \rightarrow O(c)$. Moreover, the action of $K$ on $K / A$ sends fibers $S^{b-1}$ isometrically to other fibers $S^{b-1}$, for the standard metric on the sphere, and likewise for the other spheres $S^{c-1}$. The principal $O(b)$-bundle over $K / B$ corresponding to the sphere bundle $S^{b-1} \rightarrow K / A \rightarrow K / B$ is $(K \times O(b)) / B$, which has an $O(b)$-equivariant good complexification by Lemma 3.1, and the same comments apply to the sphere bundle $S^{c-1} \rightarrow K / A \rightarrow K / C$.

It is straightforward to check that the antipodal map on each fiber of the sphere bundle $S^{b-1} \rightarrow K / A \rightarrow K / B$ is a $K$-equivariant map $K / A \rightarrow K / A$, and the same goes for the other sphere bundle with total space $K / A$. But the set of $K$-maps from $K / A$ to itself is the group $N_{K}(A) / A$, acting on $K / A$ by $g A \mapsto g b A$ for $b \in N_{K}(A)$. Explicitly, the first $\mathbf{Z} / 2$-action on $K / A$ is described by the image in $N_{K}(A) / A$ of any element $b \in B$ which takes the point $1 \cdot A \in B / A \cong S^{b-1}$ to its antipode, and the second $\mathbf{Z} / 2$-action has the analogous description in terms of $C$. Thus, although the two $\mathbf{Z} / 2$-actions on $K / A$ coming from the two sphere bundles need not be the same, they are both contained in the compact Lie group $N_{K}(A) / A$ which acts on $K / A$ and commutes with the action of the compact Lie group $K$. So the Riemannian metric on $K / A$ associated to a bi-invariant metric 
on $K$ is preserved by both $\mathbf{Z} / 2$-actions as well as the action of $K$. By Theorem 5.1, $M$ has a good complexification with an action of the complexification $G$ of $K$.

Proof of Theorem 5.1. This theorem is a direct generalization of Theorem 4.1, and we will imitate the simpler proof of Theorem 4.1 as far as possible.

We are given that the two $\mathbf{Z} / 2$-actions on the closed manifold $E(\mathbf{R})$ map into the compact Lie group $\operatorname{Isom}(E(\mathbf{R}))$ of isometries of $E(\mathbf{R})$ for some metric. We will show that we can arrange for the two copies of $\mathbf{Z} / 2$ to generate only a finite dihedral subgroup of $\operatorname{Isom}(E(\mathbf{R}))$.

We know that the image of the corresponding homomorphism $\mathbf{Z} / 2 * \mathbf{Z} / 2 \rightarrow$ $\operatorname{Isom}(E(\mathbf{R}))$ has compact closure $K$. Here the free product $\mathbf{Z} / 2 * \mathbf{Z} / 2$ is the infinite dihedral group, and in particular it has an abelian subgroup $\mathbf{Z}$ of index 2 . So the identity component of the compact Lie group $K$ is a torus $T$.

Let $\beta$ and $\gamma$ denote the generators of the two copies of $\mathbf{Z} / 2$ in $K$. Let $n$ be a positive integer such that $(\beta \gamma)^{n}$ is in $T$. Clearly $(\beta \gamma)^{\text {in }}$ topologically generates $T$ for each positive integer $i$; otherwise the identity component of $K$ would be smaller than $T$. Now an explicit calculation in $\mathbf{Z} / 2 * \mathbf{Z} / 2$ shows that for any integer $k$, if we let $\alpha=(\beta \gamma)^{k}$, then

$$
\left(\beta \alpha \gamma \alpha^{-1}\right)^{n}=(\beta \gamma)^{n-2 n k} .
$$

Since $(\beta \gamma)^{2 n^{2}}$ topologically generates the torus $T$, we can find a sequence of integers $k_{i}$ such that $(\beta \gamma)^{2 n^{2} k_{i}}$ approaches $(\beta \gamma)^{n}$. Then, if we let $\alpha$ be the limit of some subsequence of the elements $(\beta \gamma)^{n k_{i}}$ of the compact torus $T$, we have

$$
\left(\beta \alpha \gamma \alpha^{-1}\right)^{n}=1 \text {. }
$$

That is, by replacing $\gamma$ by $\alpha \gamma \alpha^{-1}$, we can arrange that $\beta$ and $\gamma$ generate only a finite dihedral subgroup of $K$. This change corresponds to changing our identification of the total spaces of the two sphere bundles $S^{b-1} \rightarrow E(\mathbf{R}) \rightarrow B(\mathbf{R})$ and $S^{c-1} \rightarrow E(\mathbf{R}) \rightarrow C(\mathbf{R})$ by the automorphism $\alpha$ of $E(\mathbf{R})$. We chose $\alpha$ to lie in the torus $T$, thus in the identity component of the isometry group of $E$, and so this change of identification does not change the diffeomorphism type of the manifold $M$ obtained by gluing the two disc bundles together. In short, to find a good complexification of $M$, we can assume that the two $\mathbf{Z} / 2$-actions generate only a finite dihedral subgroup

$$
D_{2 n}=\left\langle\beta, \gamma: \beta^{2}=\gamma^{2}=(\beta \gamma)^{n}=1\right\rangle
$$

of the isometry group of $E(\mathbf{R})$.

Even with our description of $M$ thus simplified, we cannot in general define the real algebraic variety $U$ we want by gluing together Zariski open subsets of the varieties $U_{B}$ and $U_{C}$ considered in the proof of Theorem 4.1 (which concerns the special case $n=1$, where the two $\mathbf{Z} / 2$-actions on $E$ are the same). Instead, we will define $U$ by a more sophisticated kind of gluing, Grothendieck's theorem on faithfully flat descent. The theorem is easy to use, despite the forbidding name. See Grothendieck [10] for a survey and Waterhouse [24], pp. 132-133, for a short proof. 
Here is Grothendieck's theorem in the case we need. Let $f: L \rightarrow A$ be a faithfully flat morphism of affine schemes. For example, this is true if $L$ and $A$ are smooth affine varieties over a field, $f$ is etale (the derivative is an isomorphism at every point of $L$ ), and $f$ is surjective; all of this will be true in the case we need. Consider an affine scheme $N$ with a map $g: N \rightarrow L$. Define a descent datum on $N \rightarrow L$, from $L$ down to $A$, to be an isomorphism $f: N \times_{A} L \rightarrow L \times_{A} N$ of schemes over $L \times_{A} L$, thus an isomorphism from $g^{-1}\left(l_{1}\right)$ to $g^{-1}\left(l_{2}\right)$ for all pairs of points $l_{1}, l_{2} \in L$ with the same image in $A$, which satisfies the cocycle condition that for any three points $l_{1}, l_{2}, l_{3}$ in $L$ with the same image in $A$, the composition

$$
g^{-1}\left(l_{1}\right) \rightarrow g^{-1}\left(l_{2}\right) \rightarrow g^{-1}\left(l_{3}\right)
$$

is the given isomorphism $g^{-1}\left(l_{1}\right) \rightarrow g^{-1}\left(l_{3}\right)$.

Theorem 5.3 (Grothendieck). Let $L \rightarrow$ A be a faithfully flat morphism of affine schemes. Then there is an equivalence of categories from affine schemes $M \rightarrow A$ to affine schemes $N \rightarrow L$ together with a descent datum from $L$ to $A$. The equivalence takes $M$ to $N:=M \times_{A}$ L, which has an obvious descent datum.

For example, if we write the affine line $A=A^{1}$ as a union of two affine open subsets $S$ and $T$, then the map from the disjoint union $S \bigsqcup T$ to $A$ is etale and surjective, hence faithfully flat, and the theorem allows us to construct an affine variety $U \rightarrow A^{1}$ starting from affine varieties over $S$ and over $T$; the descent datum in that case is simply an isomorphism between the two inverse images of $S \cap T$. Thus the theorem specializes to the construction of affine varieties by gluing Zariski open subsets, as in the proof of Theorem 4.1.

In the case at hand, we have to consider a more complicated faithfully flat map to $A_{\mathbf{R}}^{1}$. First, consider the standard action of the dihedral group $D_{2 n}=\left\langle\beta, \gamma: \beta^{2}=\right.$ $\left.\gamma^{2}=(\beta \gamma)^{n}=1\right\rangle$ on the real algebraic curve $S^{1}$, defined by

$$
\begin{aligned}
& \beta(z)=\zeta_{n} z^{-1} \\
& \gamma(z)=z^{-1} .
\end{aligned}
$$

Here we view roots of unity such as $\zeta_{n}=\exp (2 \pi i / n)$ as elements of the group $S^{1}(\mathbf{R})$. The geometric quotient $S^{1} / D_{2 n}$ is isomorphic to the affine line $A_{\mathbf{R}}^{1}$. Explicitly, we will use the isomorphism given on $z \in S^{1} \subset \mathbf{C}^{*}$ by $z \mapsto \operatorname{Re} z^{n}$. The dihedral group acts freely on $S^{1}$ outside the $(2 n)$ th roots of unity, and it has two orbits on the $(2 n)$ th roots of unity, the points $\zeta_{2 n}^{i}$ with $i$ even and the points $\zeta_{2 n}^{i}$ with $i$ odd.

Define affine curves $L_{B}$ and $L_{C}$ over $\mathbf{R}$ as the geometric quotients

$$
\begin{aligned}
& L_{B}=\left(S^{1}-\left\{\zeta_{2 n}^{i}: i \not \equiv 1 \quad(\bmod 2 n)\right\}\right) /\langle\beta\rangle \\
& L_{C}=\left(S^{1}-\left\{\zeta_{2 n}^{i}: i \not \equiv 0 \quad(\bmod 2 n)\right\}\right) /\langle\gamma\rangle .
\end{aligned}
$$

There are natural maps from $L_{B}$ and $L_{C}$ to $S^{1} / D_{2 n}=A_{\mathbf{R}}^{1}$. These maps are etale, since in defining $L_{B}$ we have divided out by the whole stabilizer subgroup $\langle\beta\rangle \cong \mathbf{Z} / 2$ of the point $\zeta_{2 n} \in S^{1}$, and in defining $L_{C}$ we have divided out by the 
whole stabilizer subgroup $\langle\gamma\rangle$ of the point $1 \in S^{1}$. At the same time, we see that $L_{B}$ and $L_{C}$ are smooth curves, since $A^{1}$ is a smooth curve. Finally, the image of $L_{B}$ in $A^{1}$ is $A^{1}-\{1\}$, and the image of $L_{C}$ in $A^{1}$ is $A^{1}-\{-1\}$. Let $L$ be the disjoint union of the two curves $L_{B}$ and $L_{C}$. The map $L \rightarrow A_{\mathbf{R}}^{1}$ is etale and surjective, hence faithfully flat. It will later be very convenient that the inverse images in the curve $L$ of the points -1 and 1 in the affine line are both single points.

We will define the variety $U$ we want, along with a map of it to the affine line, by faithfully flat descent from a variety $V \rightarrow L$. Here we define $V$ as the disjoint union of the following affine varieties $V_{B}$ and $V_{C}$. By the definition of sphere bundles before Theorem 4.1, we are given good complexifications $P_{B}$ and $P_{C}$ with free actions of $O(b)$ and $O(c)$ such that $E=P_{B} / O(b-1)=P_{C} / O(c-1)$, $B=P_{B} / O(b)$, and $C=P_{C} / O(c)$.

$$
\begin{aligned}
& V_{B}=\left[P_{B} \times\left(S^{b}-\left\{x_{b}=\operatorname{Re} \zeta_{2 n}^{j} \text { for some } j \not \equiv 0 \quad(\bmod 2 n)\right\}\right)\right] / O(b) \\
& V_{C}=\left[P_{C} \times\left(S^{c}-\left\{x_{c}=\operatorname{Re} \zeta_{2 n}^{j} \text { for some } j \not \equiv 0 \quad(\bmod 2 n)\right\}\right)\right] / O(c) \text {. }
\end{aligned}
$$

Since $O(b)$ acts freely on $P_{B}$ with quotient $B$, the affine variety $V_{B}$ is an open subset of an $S^{b}$-bundle over $B$ and hence is smooth. Likewise, $V_{C}$ is an open subset of an $S^{c}$-bundle over $C$ and hence is smooth.

There is an obvious map $x_{b}: V_{B} \rightarrow A^{1}$, since the orthogonal group $O(b)$ only acts on the first $b$ coordinates $\left(x_{0}, \ldots, x_{b-1}\right)$ in $S^{b}$. Likewise, we have a map $x_{c}: V_{C} \rightarrow A^{1}$. More important, we define a map $V_{B} \rightarrow L_{B}$ which sends $x_{b}$ to the image in $L_{B}$ of $\left(x_{b}, y_{b}\right) \zeta_{2 n} \in S^{1}$ for any $y_{b}$ with $x_{b}^{2}+y_{b}^{2}=1$. And we define a map $V_{C} \rightarrow L_{C}$ which sends $x_{b}$ to the image in $L_{C}$ of $\left(x_{b}, y_{b}\right) \in S^{1}$ for any $y_{b}$ with $x_{b}^{2}+y_{b}^{2}=1$. It is clear from the definitions of $L_{B}$ and $L_{C}$ as quotients of open subsets of the circle by $\mathbf{Z} / 2$ that these are well-defined real algebraic maps.

Write $V$ for the disjoint union of $V_{B}$ and $V_{C}$, and $L$ for the disjoint union of $L_{B}$ and $L_{C}$. In order to define the variety $U \rightarrow A_{\mathbf{R}}^{1}$ that we want, we have to define a descent datum for the variety $V \rightarrow L$ from $L$ to $A_{\mathbf{R}}^{1}$. Here we are using the map $L \rightarrow A_{\mathbf{R}}^{1}$ defined earlier. By definition, as discussed above, a descent datum is an isomorphism $f: V \times_{A^{1}} L \rightarrow L \times_{A^{1}} V$ over $L \times_{A^{1}} L$ which satisfies the cocycle condition.

Over the open subset $A^{1}-\{ \pm 1\}$, this is easy. Let us write $L_{B}^{0}, L_{C}^{0}, V_{B}^{0}, V_{C}^{0}$ for the inverse images of $A^{1}-\{ \pm 1\}$ in the varieties $L_{B}, L_{C}, V_{B}, V_{C}$. Then $V_{B}^{0}$ and $V_{C}^{0}$ have simple descriptions: they are open subsets of the varieties $U_{B}$ and $U_{C}$ considered in the previous section. Precisely, by Lemma 4.3, we have

$$
V_{B}^{0}=\left[E \times\left(S^{1}-\left\{\zeta_{2 n}^{j}: j \in \mathbf{Z}\right\}\right)\right] / \mathbf{Z} / 2,
$$

where $\mathbf{Z} / 2$ acts on $E$ by the antipodal map in the sphere bundle $S^{b-1} \rightarrow E \rightarrow B$, and by the reflection $z \mapsto z^{-1}$ on the circle. We will actually use a slightly different description of $V_{B}^{0}$. After multiplying points in the circle by $\zeta_{2 n}$, we can say that

$$
V_{B}^{0}=\left[E \times\left(S^{1}-\left\{\zeta_{2 n}^{j}: j \in \mathbf{Z}\right\}\right)\right] /\langle\beta\rangle,
$$

where $\langle\beta\rangle \cong \mathbf{Z} / 2$ acts on $E$ as above, and by the reflection $\beta(z)=\zeta_{n} z^{-1}$ on the circle. This description has the advantage that the map $V_{B} \rightarrow L_{B}$ defined earlier is simply the projection to $S^{1} /\langle\beta\rangle$. 
Likewise, Lemma 4.3 shows that

$$
V_{C}^{0}=\left[E \times\left(S^{1}-\left\{\zeta_{2 n}^{j}: j \in \mathbf{Z}\right\}\right)\right] /\langle\gamma\rangle,
$$

where $\langle\gamma\rangle \cong \mathbf{Z} / 2$ acts on $E$ by the antipodal map of the other sphere bundle $S^{c-1} \rightarrow E \rightarrow C$, and on the circle by the reflection $\gamma(z)=z^{-1}$. As in the case of $V_{B}^{0}$, the map $V_{C}^{0} \rightarrow L_{C}$ defined earlier is the obvious projection to $S^{1} /\langle\gamma\rangle$.

So we see that both $V_{B}^{0}$ and $V_{C}^{0}$ map to the variety

$$
U^{0}:=\left[E \times\left(S^{1}-\left\{\zeta_{2 n}^{j}: j \in \mathbf{Z}\right\}\right)\right] / D_{2 n} .
$$

This will be an open subset of the variety $U$ we want (which will not have such a simple description itself). The variety $U^{0}$ projects to $S^{1} / D_{2 n}=A_{\mathbf{R}}^{1}$, and there are obvious isomorphisms

$$
\begin{aligned}
& U^{0} \times{ }_{A^{1}} L_{B} \cong V_{B}^{0} \\
& U^{0} \times{ }_{A^{1}} L_{C} \cong V_{C}^{0} .
\end{aligned}
$$

So $U^{0} \times{ }_{A^{1}} L \cong V^{0}$, where we write $V^{0}$ for the disjoint union of $V_{B}^{0}$ and $V_{C}^{0}$. That is, the variety $V^{0} \rightarrow L$ is obtained from the variety $U^{0} \rightarrow A^{1}$ by pulling back from $A^{1}$ to $L$. As a result, in the terminology of Theorem 5.3, we have a natural descent datum on $V^{0} \rightarrow L$, from $L$ down to $A^{1}$.

I claim that this descent datum extends to a descent datum on $V \rightarrow L$, from $L$ down to $A^{1}$; then Theorem 5.3 will give a variety $U \rightarrow A^{1}$ which is in fact the one we want. The claim about descent data means that a certain isomorphism $f: V^{0} \times{ }_{A^{1}} L \rightarrow L \times_{A^{1}} V^{0}$ over $L \times{ }_{A^{1}} L$ extends to an isomorphism $V \times_{A^{1}} L \rightarrow$ $L \times{ }_{A^{1}} V$. (The cocycle condition for the extended map follows automatically, since $V^{0}$ is a dense open subset of $V$.)

Here $V^{0}$ is the inverse image of $A^{1}-\{ \pm 1\}$ in $V$. The curve $L$ has only one point which maps to -1 in $A^{1}$ and one point which maps to 1 in $A^{1}$. It follows that $L \times{ }_{A^{1}} L$ has only two points which do not map into $A^{1}-\{ \pm 1\}$, the point $\left(\zeta_{2 n}, \zeta_{2 n}\right) \in L_{B} \times{ }_{A^{1}} L_{B}$ which maps to -1 and the point $(1,1) \in L_{C} \times{ }_{A^{1}} L_{C}$ which maps to 1 . Both of these points belong to the diagonal subvariety $L \subset L \times{ }_{A^{1}} L$, which is an open and closed subset of $L \times{ }_{A^{1}} L$ because $L$ is etale over $A^{1}$. Therefore $V \times{ }_{A^{1}} L-V^{0} \times_{A^{1}} L$ maps into this open and closed subset $L \subset L \times_{A^{1}} L$.

The inverse image of the diagonal subset $L \subset L \times_{A^{1}} L$ in $V \times{ }_{A^{1}} L$, as in $L \times_{A^{1}} V$, is isomorphic to $V$ in a natural way. In terms of these identifications, the given descent datum restricted to the inverse image of this diagonal subset is an isomorphism $f: V^{0} \rightarrow V^{0}$. It is in fact the identity map. Indeed, the definition of a descent datum implies that the isomorphism is the identity map over the diagonal subset $L \subset L \times{ }_{A^{1}} L$. Therefore, $f$ extends to an isomorphism $V \rightarrow V$, namely the identity map. By the previous paragraph, this completes the proof that the descent datum for $V^{0} \rightarrow L$ extends to a descent datum for $V \rightarrow L$, from $L$ to $A_{\mathbf{R}}^{1}$.

By Theorem 5.3, this datum determines an affine scheme $U$ over the real numbers, with a map $U \rightarrow A_{\mathbf{R}}^{1}$ and an isomorphism from $U \times{ }_{A^{1}} L$ to $V$. Since $L \rightarrow A^{1}$ is etale and surjective and the pullback $V$ of $U$ from $A^{1}$ to $L$ is a smooth variety over $\mathbf{R}, U$ is a smooth variety over $\mathbf{R}$. 
It is clear from the construction that the inverse image of $A^{1}-\{ \pm 1\}$ in $U$ is the variety $U^{0}$ described earlier,

$$
U^{0}=\left[E \times\left(S^{1}-\left\{\zeta_{2 n}^{j}: j \in \mathbf{Z}\right\}\right)\right] / D_{2 n}
$$

By Lemma 3.1, $\left(E \times S^{1}\right) / D_{2 n}$ is a good complexification of $\left(E(\mathbf{R}) \times S^{1}\right) / D_{2 n}$, so the image of its real points in $A^{1}(\mathbf{R})$ is the interval $[-1,1]$. It follows that the map $U(\mathbf{R}) \rightarrow A^{1}(\mathbf{R})$ has image contained in the interval $[-1,1]$, and that it a fibration over $(-1,1)$ with fiber $E(\mathbf{R})$. Since $L_{B} \rightarrow A^{1}$ and $L_{C} \rightarrow A^{1}$ are etale open neighborhoods of -1 and 1 respectively, the isomorphisms $U \times{ }_{A^{1}} L_{B} \cong V_{B}$ and $U \times{ }_{A^{1}} L_{C} \cong V_{C}$ show what the map $U(\mathbf{R}) \rightarrow[-1,1]$ looks like near -1 and 1 , respectively. We see that $U(\mathbf{R}) \rightarrow[-1,1]$ is a proper map, with fibers over -1 and 1 isomorphic to $B(\mathbf{R})$ and $C(\mathbf{R})$. Clearly $U(\mathbf{R})$ is diffeomorphic to the manifold $M$ we want, the union of the two disc bundles.

We can use the same etale open neighborhoods to analyze $U(\mathbf{C})$. As in the proof of Theorem 4.1, we can check that $U(\mathbf{R}) \rightarrow U(\mathbf{C})$ is a homotopy equivalence by checking this over the open subsets

$$
S=\{z \in \mathbf{C}: \operatorname{Re} z<1 / 2\}
$$

and

$$
T=\{z \in \mathbf{C}: \operatorname{Re} z>-1 / 2\}
$$

of $A^{1}(\mathbf{C})$, as well as over the open subset $S \cap T$; the argument is exactly the same as in the proof of Theorem 4.1. Thus $U$ is a good complexification of $M$.

Finally, if we have a compact Lie group which acts compatibly on $E, B$, and $C$, satisfying the assumptions in Theorem 5.1, then its complexification $G$ acts on the real algebraic variety $U$ we have constructed. So $U$ is a $G$-equivariant good complexification of $M$.

$\square($ Theorem 5.1)

\section{Uniqueness of good complexifications for $S^{1}, S^{2}$, and $\mathbf{R} \mathbf{P}^{2}$}

In this section we prove that $S^{1}$ has a unique good complexification. More interestingly, $S^{2}$ and $\mathbf{R P}^{2}$ each have a unique good complexification $U$ with a certain extra property: $U(\mathbf{C})$ is diffeomorphic (or just proper homotopy equivalent) to the total space of the tangent bundle of $U(\mathbf{R})$. Notice that for any smooth algebraic variety $U$ over $\mathbf{R}$, the normal bundle of $U(\mathbf{R})$ in $U(\mathbf{C})$ is isomorphic to the tangent bundle of $U(\mathbf{R})$ as a real vector bundle. So if the pair $(U(\mathbf{R}), U(\mathbf{C}))$ is diffeomorphic to the total space of any vector bundle over $U(\mathbf{R})$, it must be diffeomorphic to the total space of the tangent bundle of $U(\mathbf{R})$.

This kind of uniqueness seems unlikely in higher dimensions, since topological information has stronger consequences for algebraic surfaces than for higherdimensional varieties.

Theorem 6.1. For $M=S^{1}$, there is exactly one isomorphism class of smooth real quasi-projective varieties $U$ such that $U(\mathbf{R})$ is diffeomorphic to $M$ and the inclusion $U(\mathbf{R}) \rightarrow U(\mathbf{C})$ is a homotopy equivalence. For $M=S^{2}$ and $\mathbf{R P}^{2}$, there is exactly 
one isomorphism class of smooth real quasi-projective varieties $U$ such that $U(\mathbf{R})$ is diffeomorphic to $M$, the map $U(\mathbf{R}) \rightarrow U(\mathbf{C})$ is a homotopy equivalence, and $U(\mathbf{C})$ is proper homotopy equivalent to the total space of the tangent bundle of $M=U(\mathbf{R})$.

Proof. Since $S^{n}$ and $\mathbf{R P}^{n}$ are compact homogeneous Riemannian manifolds, they have good complexifications. Explicitly, for $S^{n}$ we have the affine hypersurface

$$
U=\left\{x_{0}^{2}+\cdots+x_{n}^{2}=1\right\} \subset A_{\mathbf{R}}^{n+1},
$$

and for $\mathbf{R} \mathbf{P}^{n}, U$ is the complement of the divisor $D=\left\{x_{0}^{2}+\cdots+x_{n}^{2}=0\right\}$ in $\mathbf{P}_{\mathbf{R}}^{n}$.

For $S^{1}$, the uniqueness is easy. Let $U$ be a smooth real algebraic curve with $U(\mathbf{R})$ diffeomorphic to the circle such that $U(\mathbf{R}) \rightarrow U(\mathbf{C})$ is a homotopy equivalence. Then the first Betti number of $U(\mathbf{C})$ is 1 . Let $X$ be a smooth compactification of $U$ defined over $\mathbf{R}$, so that $U(\mathbf{C})=X(\mathbf{C})-\{k$ points $\}$ for some $k \geq 1$. Then $1=b_{1}(U(\mathbf{C}))=b_{1}(X(\mathbf{C}))+k-1$, and $b_{1}(X)=2 g$ where $g$ is the genus of $X$, so $g=0$ and $k=2$. That is, $X \times_{\mathbf{R}} \mathbf{C}$ is isomorphic to $\mathbf{P}_{\mathbf{C}}^{1}$, and $U \times{ }_{\mathbf{R}} \mathbf{C} \cong A_{\mathbf{R}}^{1}-0$. There are two real forms of $\mathbf{P}_{\mathbf{C}}^{1}, \mathbf{P}_{\mathbf{R}}^{1}$ and a conic with no real points. In this case, $X(\mathbf{R})$ is nonempty, so $X$ is isomorphic to $\mathbf{P}_{\mathbf{R}}^{1}$. We know that $U(\mathbf{R})=X(\mathbf{R})$ since $X(\mathbf{R})$ is a 1-manifold by smoothness of $X$ and since $U(\mathbf{R})$ is a closed 1-manifold. So $U$ is the complement of two conjugate complex points in $\mathbf{P}_{\mathbf{R}}^{1}$, rather than two real points. The automorphism group $P G L(2, \mathbf{R})$ of $\mathbf{P}_{\mathbf{R}}^{1}$ acts transitively on the set of pairs of conjugate complex points (equivalently, on the upper half-plane), so $U$ is isomorphic to $\mathbf{P}_{\mathbf{R}}^{1}-\{i,-i\}$. Equivalently, $U$ is isomorphic to the real affine curve

$$
S^{1}=\left\{(x, y) \in A_{\mathbf{R}}^{2}: x^{2}+y^{2}=1\right\} .
$$

Now let $M=S^{2}$. Let $U$ be a good complexification of $M$ with the stronger assumption made in the theorem. Let $X$ be a smooth projective compactification of $U$ defined over $\mathbf{R}$ such that $D:=X-U$ is a divisor with simple normal crossings ("simple" means that each irreducible component of $D$ is smooth) in the algebraic surface $X$. Since $\chi(M)>0$, Theorem 2.1 shows that the weight filtration on $H^{i}(U(\mathbf{C}), \mathbf{Q})$ is in weight $i$ for all $i$. Equivalently, by the definition of the weight filtration, the restriction map $H^{*}(X(\mathbf{C}), \mathbf{Q}) \rightarrow H^{*}(U(\mathbf{C}), \mathbf{Q})$ is surjective.

From the exact sequence of Borel-Moore homology,

$$
H_{i} D(\mathbf{C}) \rightarrow H_{i} X(\mathbf{C}) \rightarrow H_{i}^{\mathrm{BM}} U(\mathbf{C}) \rightarrow H_{i-1} D(\mathbf{C}),
$$

where $H_{i}^{\mathrm{BM}} U(\mathbf{C})=H^{4-i} U(\mathbf{C})=H^{4-i}\left(S^{2}\right)$ and the restriction map from $X$ to $U$ is surjective with $\mathbf{Q}$ coefficients, we can read off several facts. First, $D(\mathbf{C})$ is connected since $X(\mathbf{C})$ is. Next, $H_{3}(X(\mathbf{C}), \mathbf{Q})=0$, so by Poincaré duality we also have $H_{1}(X(\mathbf{C}), \mathbf{Q})=0$. From there, the exact sequence shows that $H_{1}(D(\mathbf{C}), \mathbf{Q})=0$. We know that $D_{\mathbf{C}}:=D \times_{\mathbf{R}} \mathbf{C}$ is a union of smooth complex algebraic curves identified along nodes, and these results show that $D_{\mathbf{C}}$ is in fact a union of smooth rational curves with dual graph a tree. Let $T$ be the dual graph of $D_{\mathbf{C}}$, "weighted" by writing the self-intersection number of each irreducible component of $D_{\mathbf{C}}$ at the corresponding vertex of $T$. Also, since $D$ is defined over $\mathbf{R}$, complex conjugation gives an involution of the weighted tree $T$. 
Since we assume that $U(\mathbf{C})$ is proper homotopy equivalent to the tangent bundle of $S^{2}$, the fundamental group of $U(\mathbf{C})$ at infinity is equal to $\pi_{1}^{\infty}\left(T S^{2}\right)=\mathbf{Z} / 2$. By Ramanujam [21], the fundamental group of $U(\mathbf{C})$ at infinity is isomorphic to a group $\pi(T)$ associated to the weighted tree $T$. So we know that the weighted tree $T$ has $\pi(T) \cong \mathbf{Z} / 2$. Also, we can blow up any point of $D_{\mathbf{C}}$ on $X$ and get another compactification of $U_{\mathbf{C}}$ by a simple normal crossing divisor, and we can apply the inverse operation (blowing down a $(-1)$-curve such that the corresponding vertex in the dual graph meets at most two other vertices). These operations do not change $\pi(T)$, clearly.

As in Ramanujam, define a weighted tree $T$ to be minimal if $T$ has no vertex of degree at most 2 with weight -1 . If the weighted tree associated to a compactification $X$ of $U$ as above is not minimal, then we can find another compactification of $U$ by a simple normal crossing divisor with fewer irreducible components. This is clear over $\mathbf{C}$, by blowing down a $(-1)$-curve. To make it work over $\mathbf{R}$, we have to observe that the conjugate of any $(-1)$-curve $C \subset D$ is either disjoint from $C$ or equal to $C$, because $D(\mathbf{R})$ is empty (since $X(\mathbf{R})$ is a smooth 2-manifold and $U(\mathbf{R})$ is the closed 2-manifold $S^{2}$ ). So we can blow down $C$, or $C$ together with its conjugate, over $\mathbf{R}$. Applying this fact repeatedly, we find that the real algebraic surface $U$ has a compactification $X$ by a simple normal crossing divisor $D$ such that the associated weighted tree is minimal. We will show that under these conditions, $T$ must be a single vertex.

By Gurjar and Shastri [13], Theorem 1, p.467, any minimal weighted tree satisfying certain conditions $(\mathrm{E})$ and $(\mathrm{H})$ such that the associated group $\pi(T)$ is isomorphic to $\mathbf{Z} / 2$ is linear. The conditions $(\mathrm{E})$ and $(\mathrm{H})$ hold for a tree $T$ as above. Namely, (E) says that every linear subspace of $H_{2}(X(\mathbf{C}), \mathbf{R})$ which is positive definite for the intersection form has dimension at most 1 , which follows from the Hodge index theorem on $X_{\mathbf{C}}$. And $(\mathrm{H})$ holds for any normal crossing arrangement of rational curves on a complex surface with $b_{1}(X(\mathbf{C}))=0$ ([13], p.463). As a result, the weighted tree $T$ associated to a compactification $X$ of $U$ as in the previous paragraph is linear.

The abelianization of $\pi(T)$ has finite order if and only if the discriminant $\operatorname{disc}(T)$ of the intersection form on $H_{2}(D(\mathbf{C}), \mathbf{Z})$ is nonzero, and in that case the absolute value of $|\operatorname{disc}(T)|$ is equal to the order of the abelianization of $\pi(T)$. In our case, $\pi(T)=\mathbf{Z} / 2$ and so $|\operatorname{disc}(T)|=2$. So the intersection form on $H_{2}(D(\mathbf{C}), \mathbf{R})$ is nondegenerate, with either zero- or one-dimensional positive part by the Hodge index theorem. Also, since $|\operatorname{disc}(T)|=2$, if $T$ is a single vertex then it has weight \pm 2 . Suppose that $T$ consists of more than one vertex; we will derive a contradiction.

We gain some leverage from the fact that the divisor $D$ is defined over $\mathbf{R}$, so that complex conjugation gives an involution on the tree $T$. Moreover $D(\mathbf{R})$ is empty (as usual, because $U(\mathbf{R})=S^{2}$ is a closed 2-manifold and $X(\mathbf{R})$ must also be a 2 -manifold). It follows that the involution does not preserve any edge in the tree $T$, since that would give a singular point of $D$ which was defined over R. So conjugation acts by reversing the linear tree $T$, not as the identity. Also, the number of vertices $k$ of $T$ must be odd, so that there is a central vertex of $T$ mapped to itself by conjugation; if $k$ were even, there would be a central edge of $T$ mapped 
to itself by conjugation, contrary to what we have shown. We have $k \geq 3$ since we are assuming that $T$ is not just a single vertex.

We know that the intersection form on $H_{2}(D(\mathbf{C}), \mathbf{R})$ is nondegenerate, with at most one positive direction and the rest negative. So $T$ cannot contain two nonadjacent vertices of nonnegative weight, since they would span a 2-dimensional subspace of $H_{2}(D(\mathbf{C}), \mathbf{R})$ on which the intersection form was nonnegative. Since conjugation preserves the weights of $T, T$ must have weights $\leq-2$ outside the central vertex (using that $T$ is minimal, so there are no -1 weights). Now we are done by Gurjar-Shastri's Lemma 7, p. 467, which says that there is no minimal linear tree $T$ with $\pi_{1}(T)=\mathbf{Z} / 2$ and with all weights $\leq-2$ except for a single vertex which is not an endpoint of the tree [13]. So $T$ must be a single vertex of weight \pm 2 .

We have now almost completed the proof of Theorem 6.1 for $M=S^{2}$. We have shown that the real algebraic surface $U$ has a compactification by a smooth projective surface $X$ such that the complement $D$ is a smooth rational curve of self-intersection \pm 2 . It follows from the long exact sequence of Borel-Moore homology for $D(\mathbf{C}), X(\mathbf{C})$, and $U(\mathbf{C})$ that $b_{2}(X(\mathbf{C}))=2$. Suppose that $D$ has selfintersection -2 ; we will derive a contradiction. In this case, the canonical bundle $K_{X}$ satisfies $K_{X} \cdot D(\mathbf{C})=0$ since $D$ is a $(-2)$-curve and also $K_{X} \cdot S^{2}=0$, where $S^{2}=X(\mathbf{R})$, since the complex tangent bundle of $X$ restricted to $X(\mathbf{R})$ is isomorphic to $T S^{2} \otimes_{\mathbf{R}} \mathbf{C}$, so that $\left.K_{X}^{\otimes 2}\right|_{X(\mathbf{R})}$ is trivial. But $H^{2}(X(\mathbf{C}), \mathbf{Q})$ is generated by $D(\mathbf{C})$ and $S^{2}$, as one easily checks from the exact sequence relating the Borel-Moore homology of $X(\mathbf{C}), D(\mathbf{C}) \cong \mathbf{P}^{1}$, and $U(\mathbf{C}) \simeq S^{2}$. So $K_{X}=0$ in $H^{2}(X(\mathbf{C}), \mathbf{Q})$. In fact, $H^{1}(X(\mathbf{C}), \mathbf{Z})=0$ and $H^{2}(X(\mathbf{C}), \mathbf{Z}) \cong \mathbf{Z}^{2}$ is torsion-free, by the same exact sequence, so the line bundle $K_{X}$ is trivial. These conditions say that $X_{\mathbf{C}}$ is a K3 surface, and in particular that $b_{2}(X(\mathbf{C}))=22$ by the Riemann-Roch theorem ([9], p. 590). This is a contradiction. So in fact $D$ is a smooth rational curve of self-intersection 2 , not -2 .

To identify $X$, first over $\mathbf{C}$, we will embed $X$ in projective space using the linear system of $D$ on $X$. We have an exact sequence of sheaves on $X$,

$$
0 \rightarrow O \rightarrow O(D) \rightarrow N_{D / X} \rightarrow 0,
$$

where the normal bundle $N_{D / X}$ is the line bundle $O(2)$ on $D_{\mathbf{C}} \cong \mathbf{P}^{1}$. Since we showed early on that $H^{1}(X(\mathbf{C}), \mathbf{Q})=0$, we have $H^{1}\left(X_{\mathbf{C}}, O\right)=0$, so the long exact sequence of cohomology becomes a short exact sequence

$$
0 \rightarrow H^{0}\left(X_{\mathbf{C}}, O\right) \rightarrow H^{0}\left(X_{\mathbf{C}}, O(D)\right) \rightarrow H^{0}\left(\mathbf{P}^{1}, O(2)\right) \rightarrow 0 .
$$

Since $H^{0}\left(\mathbf{P}^{1}, O(2)\right)$ has dimension $3, H^{0}\left(X_{\mathbf{C}}, O(D)\right)$ has dimension 4. Moreover, this linear system is base-point-free on $X$, by the above exact sequence together with the base-point-freeness of $O(2)$ on $D_{\mathbf{C}} \cong \mathbf{P}^{1}$. So the line bundle $O(D)$ gives a morphism

$$
f: X \rightarrow \mathbf{P}^{3}
$$

with $f^{*} O(1)=O(D)$. This morphism is defined over $\mathbf{R}$, even though we worked over $\mathbf{C}$ to analyze it. By construction, the image of $f$ is nondegenerate (not contained in a hyperplane). 
Since $D^{2}=2$ on $X, f$ is either a degree-2 map onto a hyperplane or a birational morphism onto a quadric surface in $\mathbf{P}^{3}$; since $f$ is nondegenerate, it must be a birational morphism onto a quadric surface $f(X)$. Since $f(X)$ is clearly irreducible, it is either the projective cone over a smooth conic curve or else a smooth quadric surface. If $f(X)$ is the cone over a smooth conic curve, then $f$ factors through a birational morphism from $X$ to the blow-up of this cone at the singular point, which is a $\mathbf{P}^{1}$-bundle over a real conic. But we know the Betti number $b_{2}(X(\mathbf{C}))=2$, by the long exact sequence relating $X(\mathbf{C})$ to $U(\mathbf{C})$ and $D(\mathbf{C})$, whose Betti numbers we know. So $X$ is in fact isomorphic to (rather than a blow-up of) either a smooth quadric surface over $\mathbf{R}$ or a $\mathbf{P}^{1}$-bundle over a real conic curve. Also, the curve $D$ is a hyperplane section of the quadric surface, or a section of the $\mathbf{P}^{1}$-bundle.

In the second case, since $D(\mathbf{R})$ is empty, the base conic curve also has no real points, and so $X(\mathbf{R})$ is empty, contradicting our knowledge that $X(\mathbf{R})=S^{2}$. So $X$ must be a smooth quadric surface in $\mathbf{P}_{\mathbf{R}}^{3}$, and $D$ is a smooth hyperplane section in $X$.

There are three isomorphism classes of smooth quadric surfaces over $\mathbf{R}$, corresponding to quadratic forms on $\mathbf{R}^{4}$ of signature $(4,0)$ or $(0,4),(3,1)$ or $(1,3)$, and $(2,2)$. The sets of real points in the three cases are the empty set, $S^{2}$, and $S^{1} \times S^{1}$. In our case, we have $X(\mathbf{R})=S^{2}$, so the quadric surface $X$ is isomorphic over $\mathbf{R}$ to the one defined by

$$
x_{1}^{2}+x_{2}^{2}+x_{3}^{2}=x_{0}^{2}
$$

in $\mathbf{P}_{\mathbf{R}}^{3}$. There are two isomorphism classes of smooth hyperplane sections of this surface $X$ over $\mathbf{R}$, one with no real points and one with real points $=S^{1}$. Since $D(\mathbf{R})$ is empty, we have determined the isomorphism class of $X$ and $D$ over $\mathbf{R}$, and thus we know $U$ up to isomorphism. Namely,

$$
U \cong S^{2}:=\left\{x_{1}^{2}+x_{2}^{2}+x_{3}^{2}=1\right\} \subset A_{\mathbf{R}}^{3} .
$$

Thus Theorem 6.1 is proved for $M=S^{2}$.

Finally, let us prove Theorem 6.1 for $\mathbf{R P}^{2}$ in place of $S^{2}$. We use that a good complexification of $\mathbf{R} \mathbf{P}^{2}$ is doubly covered by a good complexification of $S^{2}$, which under the assumptions of the theorem must be isomorphic to the above real variety $S^{2}$. So it suffices to classify the possible free $\mathbf{Z} / 2$-actions on the above variety $S^{2}$. That follows easily from the following result.

Theorem 6.2. The automorphism group of the affine variety

$$
S^{2}=\left\{x_{1}^{2}+x_{2}^{2}+x_{3}^{2}=1\right\} \subset A_{\mathbf{R}}^{3}
$$

over the real numbers is the orthogonal group $O(3)$.

By contrast, many other good complexifications have noncompact automorphism group. See the examples in Sect. 7.

Proof of Theorem 6.2. Think of $U:=S^{2}$ as an open subset of the smooth projective quadric surface

$$
X:=\left\{x_{1}^{2}+x_{2}^{2}+x_{3}^{2}=x_{0}^{2}\right\} \subset \mathbf{P}_{\mathbf{R}}^{3} .
$$


Over $\mathbf{C}, X$ becomes isomorphic to $\mathbf{P}^{1} \times \mathbf{P}^{1}$. Complex conjugation $\sigma$ on $X$ switches the two families of lines on $X_{\mathbf{C}}$. Moreover, conjugation has no fixed points on the conic curve $D=X-U$.

Let $f$ be any automorphism of the real affine surface $U$. Let $L_{1}$ be any line on $X_{\mathbf{C}}$, and let $L_{2}=\sigma\left(L_{1}\right)$, which is a line in the other family of lines on $X_{\mathbf{C}}$. Consider the images of $L_{1}$ and $L_{2}$ under $f$; their closures $\overline{f\left(L_{1}\right)}$ and $\overline{f\left(L_{2}\right)}$ in $X_{\mathbf{C}}$ are conjugate closed curves. Since $f\left(L_{1}\right) \cong L_{1} \cong A_{\mathbf{C}}^{1}$, the closed curves $\overline{f\left(L_{i}\right)}$ have only one point each on the curve at infinity, $D(\mathbf{C})=X(\mathbf{C})-S^{2}(\mathbf{C})$. Clearly the point $D \cap \overline{f\left(L_{1}\right)}$ is conjugate to the point $D \cap \overline{f\left(L_{2}\right)}$. Since $D$ has no real points, it follows that these two points are distinct. That is, $\overline{f\left(L_{1}\right)}$ and $\overline{f\left(L_{2}\right)}$ intersect only in the open subset $S_{\mathbf{C}}^{2} \subset X_{\mathbf{C}}$, where they intersect transversely in one point because $L_{1}$ and $L_{2}$ intersect transversely in one point. So we know the intersection number on $X_{\mathbf{C}}$ :

$$
\overline{f\left(L_{1}\right)} \cdot \sigma\left(\overline{f\left(L_{1}\right)}\right)=1 .
$$

But $\operatorname{Pic}\left(X_{\mathbf{C}}\right) \cong \mathbf{Z} L_{1} \oplus \mathbf{Z} L_{2}$, so $\overline{f\left(L_{1}\right)}=a L_{1}+b L_{2}$ in $\operatorname{Pic}(X)$ for some $a, b \in \mathbf{Z}$, where $a, b \geq 0$ since $\overline{f\left(L_{1}\right)}$, like any curve on $X_{\mathbf{C}}$, has nonnegative intersection number with $L_{1}$ and $L_{2}$. So

$$
\begin{aligned}
\overline{f\left(L_{1}\right)} \cdot \sigma\left(\overline{f\left(L_{1}\right)}\right) & =\left(a L_{1}+b L_{2}\right) \cdot \sigma\left(a L_{1}+b L_{2}\right) \\
& =\left(a L_{1}+b L_{2}\right)\left(a L_{2}+b L_{1}\right) \\
& =a^{2}+b^{2} .
\end{aligned}
$$

Thus $a^{2}+b^{2}=1$. Since $a, b \geq 0$, we deduce that $\overline{f\left(L_{1}\right)}$ is linearly equivalent to either $L_{1}$ or $L_{2}$. It follows that $\overline{f\left(L_{1}\right)}$ is one of the two types of lines on $X_{\mathbf{C}}$. That is, any automorphism $f$ of the real affine surface $S^{2}$ sends lines (over $\mathbf{C}$ ) to lines.

That essentially completes the proof. Namely, we now know that $f$ acts on the set $\mathbf{P}_{\mathbf{C}}^{1} \bigsqcup \mathbf{P}_{\mathbf{C}}^{1}$ of lines on $X_{\mathbf{C}}$, which shows (by intersecting lines) how to extend $f$ to an automorphism of the projective surface $X_{\mathbf{C}}$. This automorphism of $X_{\mathbf{C}}$ is defined over $\mathbf{R}$ since the action on the open subset $S_{\mathbf{C}}^{2}$ of $X_{\mathbf{C}}$ is defined over $\mathbf{R}$. Thus $\operatorname{Aut}\left(S^{2}\right)$ is contained in $\operatorname{Aut}(X)=O(3,1) /\{ \pm 1\}$. Since $D=X-S^{2}$ is a hyperplane section of the quadric surface $X$ on which the quadratic form is definite, we have $\operatorname{Aut}\left(S^{2}\right)=O(3)$.

From there, we can easily prove Theorem 6.1 for $\mathbf{R P}^{2}$. Namely, by Theorem 6.2 , an action of $\mathbf{Z} / 2$ on the real affine surface $S^{2}$ corresponds to a homomorphism $\mathbf{Z} / 2 \rightarrow O(3)$, thus to a 3-dimensional real representation of $\mathbf{Z} / 2$. It is easy to check that the only such homomorphism that gives a free action of $\mathbf{Z} / 2$ on $S^{2}(\mathbf{R})=S^{2}$ is the antipodal map. Now, any complexification of $\mathbf{R P}^{2}$ satisfying the hypotheses of Theorem 6.1 is doubly covered by a complexification of $S^{2}$ satisfying the same hypotheses, hence, as we have shown, by the real variety $S^{2}$. So this complexification of $\mathbf{R P}^{2}$ must be the quotient of the variety $S^{2}$ by the antipodal map. This quotient is the complexification of $\mathbf{R P}^{2}$ we want:

$$
U=\mathbf{P}_{\mathbf{R}}^{2}-\left\{x_{0}^{2}+x_{1}^{2}+x_{2}^{2}=0\right\} .
$$




\section{Automorphisms}

Theorem 6.2 says that the automorphism group of the real affine variety $S^{2}$ is the orthogonal group $O(3)$. Since the Lempert-Szőke-Guillemin-Stenzel construction constructs a complexification of a closed manifold $M$ from a suitable riemannian metric, one might hope that all automorphisms of the complexification come from isometries of a suitable metric on $M$, which would imply that the automorphism group of the complexification is compact. The purpose of this section is to give counterexamples to most statements of that kind. I am grateful to Róbert Szôke for allowing me to include one of his constructions in this section.

There are several different things one might mean by the automorphism group of a complexification $U_{\mathbf{C}}$, assuming that $U_{\mathbf{C}}$ has an algebraic structure. One might consider either analytic or algebraic automorphisms of $U_{\mathbf{C}}$, and one might or might not consider only automorphisms which commute with complex conjugation (equivalently, which map the real manifold $U(\mathbf{R}) \subset U(\mathbf{C})$ into itself). This gives four groups associated to $U(\mathbf{R}) \subset U(\mathbf{C})$. Theorem 6.2 says that the group of algebraic automorphisms of the affine quadric $S_{\mathbf{C}}^{2}$ which commute with conjugation is compact, and our first goal is to show that the other three groups associated to $S^{2}$ are noncompact, in fact infinite-dimensional.

First, we show that the group of algebraic automorphisms of $S_{\mathbf{C}}^{2}$ is infinitedimensional. Here $S_{\mathbf{C}}^{2}$ is the hypersurface $a^{2}+b^{2}+c^{2}=1$ in $\mathbf{C}^{3}$. In new coordinates $x=a+i b, y=a-i b, z=c$, we can view $S_{\mathbf{C}}^{2}$ as the hypersurface $x y+z^{2}=1$. Then the additive group of polynomials $f(x)$ in one variable acts on $S_{\mathbf{C}}^{2}$ by

$$
(x, y, z) \mapsto\left(x, y-2 z f(x)-x f(x)^{2}, z+x f(x)\right) .
$$

So the group of algebraic automorphisms of $S_{\mathbf{C}}^{2}$ is infinite-dimensional.

Next, we give Szóke's proof, answering a question in his paper [23], that the group of analytic automorphisms of $S_{\mathbf{C}}^{2}$ which commute with complex conjugation is also infinite-dimensional. (Of course, either this or the previous result implies that the group of all analytic automorphisms of $S_{\mathbf{C}}^{2}$ is infinite-dimensional.) We again view $S_{\mathbf{C}}^{2}$ as the hypersurface $x y+z^{2}=1$ in $\mathbf{C}^{3}$. Complex conjugation $\sigma$ acts in these coordinates by $\sigma(x, y, z)=(\bar{y}, \bar{x}, \bar{z})$. For any entire function $g(z)$ in one variable, define

$$
F_{g}(x, y, z)=\left(x e^{i g(z)}, y e^{-i g(z)}, z\right) .
$$

This is an analytic automorphism of $\mathbf{C}^{3}$ which preserves the quadric $S_{\mathbf{C}}^{2}=\{x y+$ $\left.z^{2}=1\right\}$. In this way, the additive group of entire functions in one variable acts on $S_{\mathbf{C}}^{2}$. One checks that $F_{g}$ commutes with complex conjugation $\sigma$ if and only if $g(z)=\overline{g(\bar{z})}$, that is, the power series $g(z)=\sum a_{i} z^{i}$ has real coefficients. So the additive group of entire functions in one variable with real coefficients acts analytically on $\left(S_{\mathbf{C}}^{2}, \sigma\right)$. Thus the automorphism group of $\left(S_{\mathbf{C}}^{2}, \sigma\right)$ is infinitedimensional as claimed.

So the most one could hope for more general good complexifications $U(\mathbf{R}) \subset$ $U(\mathbf{C})$ is that the group of algebraic automorphisms of $\left(U_{\mathbf{C}}, \sigma\right)$, or equivalently of the real algebraic variety $U$, is compact. This turns out to be false. An easy example 
is that the automorphism group of the real affine variety $S^{1} \times S^{1}$ contains $G L(2, \mathbf{Z})$ and so is noncompact. Since the automorphism group of the real affine variety $S^{2}$ is compact (Theorem 6.2), one might guess that the automorphism group of the real algebraic variety $U$ should be compact at least when $U(\mathbf{R})$ has positive Euler characteristic.

But this fails as well: the automorphism group of the real affine variety $S^{2} \times S^{2}$ is noncompact. It suffices to show that the space of real algebraic maps $S^{2} \rightarrow S O$ (3) is noncompact, using the action of $S O(3)$ on $S^{2}$ (where we think of $S^{2} \times S^{2}$ as fibered over $S^{2}$, with $S O(3)$ acting on the fibers). Indeed, that space of maps contains the map

$$
S^{2} \hookrightarrow S^{3} \rightarrow S^{3} \rightarrow S O(3)
$$

for any integer $n$. Here $S^{2} \rightarrow S^{3}$ is an inclusion which takes some point $p \in S^{2}$ to the identity element for the group structure on $S^{3}$, the map $S^{3} \rightarrow S^{3}$ is the map $x \mapsto x^{n}$ with respect to the group structure on $S^{3}$, and $S^{3} \rightarrow S O(3)$ is the standard double covering. The derivatives of these maps $S^{2} \rightarrow S O(3)$ at the point $p$ tend to infinity as $n$ goes to infinity, so the space of such maps is noncompact. Thus the automorphism group of the real algebraic variety $S^{2} \times S^{2}$ is noncompact, as promised.

Finally, it is very natural to ask whether the orthogonal group $O(n+1)$ is the whole automorphism group of the sphere $S^{n}$ as a real affine algebraic variety. This is elementary for $n=1$ and true for $n=2$ by Theorem 6.2. It would be especially interesting if the answer were yes: that would be a kind of rigidity which I think has not been observed before.

\section{References}

1. R. Aguilar: Symplectic reduction and the complex homogeneous Monge-Ampère equation. Ann. Global Anal. Geom. 19 (2001), 327-353

2. R. Aguilar, D. Burns: On the algebraicization of certain Stein manifolds. http: / / xxx. lanl.gov/abs/math. CV/ 0010287 , revised 24 Sept. (2001)

3. G. Bredon: Introduction to compact transformation groups. New York: Academic Press (1972)

4. D. Burns: Curvatures of Monge-Ampère foliations and parabolic manifolds. Ann. Math. 115 (1982), 349-373

5. J. Cheeger: Some examples of manifolds of nonnegative curvature. J. Diff. Geo. 8 (1973), 623-628

6. P. Deligne: Poids dans la cohomologie des variétés algébriques. Proceedings of the International Congress of Mathematicians (Vancouver, B.C., 1974), v. 1, 79-85. Montreal: Canad. Math. Congress (1975)

7. Y. Félix: La dichotomie elliptique-hyperbolique en homotopie rationnelle. Astérisque 176 (1989)

8. W. Greub, S. Halperin, R. Vanstone: Connections, curvature, and cohomology, v. 3. New York: Academic Press (1976)

9. P. Griffiths, J. Harris. Principles of algebraic geometry. New York: John Wiley (1978)

10. A. Grothendieck: Technique de descente et théorèmes d'existence en géométrie algébrique. I. Généralités. Descente par morphismes fidelement plats. Seminaire Bourbaki 5 (1959/1960), Exposé 190, 299-327

11. K. Grove, W. Ziller. Curvature and symmetry of Milnor spheres. Ann. Math. 151 (2000), $1-36$ 
12. V. Guillemin, M. Stenzel: Grauert tubes and the homogeneous Monge-Ampère equation. J. Diff. Geo. 34 (1991), 561-570

13. R. Gurjar, A. Shastri: The fundamental group at infinity of affine surfaces. Comment. Math. Helv. 59 (1984), 459-484

14. H. Hironaka: Resolution of singularities of an algebraic variety over a field of characteristic zero. Ann. Math. 79 (1964), I: 109-203; II: 205-326

15. W.-C. Hsiang, W.-Y. Hsiang: On compact subgroups of the diffeomorphism groups of Kervaire spheres. Ann. Math. 85 (1967), 359-369

16. R. S. Kulkarni: On complexifications of differentiable manifolds. Invent. Math. $\mathbf{4 4}$ (1978), 49-64

17. L. Lempert, R. Szőke: Global solutions of the homogeneous complex Monge-Ampère equation and complex structures on the tangent bundle of Riemannian manifolds. Math. Ann. 290 (1991), 689-712.

18. Y. Matsushima: Espaces homogènes de Stein des groupes de Lie complexes. Nagoya Math. J. 16 (1960), 205-218

19. P. Mostert: On a compact Lie group acting on a manifold. Ann. Math. 65 (1957), 447-455; errata, 66 (1957), 589

20. D. Mumford, J. Fogarty: Geometric Invariant Theory. Berlin: Springer (1982)

21. C. P. Ramanujam: A topological characterization of the affine plane as an algebraic variety. Ann. Math. 94 (1971), 69-88

22. R. Szőke: Complex structures on tangent bundles of Riemannian manifolds. Math. Ann. 291 (1991), 409-428

23. R. Szőke: Automorphisms of certain Stein manifolds. Math. Z. 219 (1995), 357-385

24. W. Waterhouse: Introduction to affine group schemes. New York: Springer (1979) 